\title{
Monte Carlo Study of Lattice Compact Quantum Electrodynamics with Fermionic Matter: The Parent State of Quantum Phases
}

\author{
Xiao Yan Xu, ${ }^{1, *}$ Yang Qi,${ }^{2-4, \dagger}$ Long Zhang, ${ }^{5}$ Fakher F. Assaad, ${ }^{6}$ Cenke Xu, ${ }^{7}$ and Zi Yang Meng, ${ }^{8,9,10,11, \$}$ \\ ${ }^{1}$ Department of Physics, Hong Kong University of Science and Technology, \\ Clear Water Bay, Hong Kong, China \\ ${ }^{2}$ Center for Field Theory and Particle Physics, Department of Physics, Fudan University, \\ Shanghai 200433, China \\ ${ }^{3}$ State Key Laboratory of Surface Physics, Fudan University, Shanghai 200433, China \\ ${ }^{4}$ Collaborative Innovation Center of Advanced Microstructures, Nanjing 210093, China \\ ${ }^{5}$ Kavli Institute for Theoretical Sciences and CAS Center for Excellence in Topological Quantum \\ Computation, University of Chinese Academy of Sciences, Beijing 100190, China \\ ${ }^{6}$ Institut für Theoretische Physik und Astrophysik, Universität Würzburg, 97074, Würzburg, Germany \\ ${ }^{7}$ Department of Physics, University of California, Santa Barbara, California 93106, USA \\ ${ }^{8}$ Beijing National Laboratory for Condensed Matter Physics and Institute of Physics, \\ Chinese Academy of Sciences, Beijing 100190, China \\ ${ }^{9}$ Department of Physics, The University of Hong Kong, China \\ ${ }^{10}$ CAS Center of Excellence in Topological Quantum Computation and School of Physical Sciences, \\ University of Chinese Academy of Sciences, Beijing 100190, China \\ ${ }^{11}$ Songshan Lake Materials Laboratory, Dongguan, Guangdong 523808, China
}

\begin{abstract}
(Received 1 August 2018; revised manuscript received 19 March 2019; published 2 May 2019)
The interplay between lattice gauge theories and fermionic matter accounts for fundamental physical phenomena ranging from the deconfinement of quarks in particle physics to quantum spin liquid with fractionalized anyons and emergent gauge structures in condensed matter physics. However, except for certain limits (for instance, a large number of flavors of matter fields), analytical methods can provide few concrete results. Here we show that the problem of compact $\mathrm{U}(1)$ lattice gauge theory coupled to fermionic matter in $(2+1) \mathrm{D}$ is possible to access via sign-problem-free quantum Monte Carlo simulations. One can hence map out the phase diagram as a function of fermion flavors and the strength of gauge fluctuations. By increasing the coupling constant of the gauge field, gauge confinement in the form of various spontaneous-symmetrybreaking phases such as the valence-bond solid (VBS) and Néel antiferromagnet emerge. Deconfined phases with algebraic spin and VBS correlation functions are also observed. Such deconfined phases are incarnations of exotic states of matter, i.e., the algebraic spin liquid, which is generally viewed as the parent state of various quantum phases. The phase transitions between the deconfined and confined phases, as well as that between the different confined phases provide various manifestations of deconfined quantum criticality. In particular, for four flavors $N_{f}=4$, our data suggest a continuous quantum phase transition between the VBS and Néel order. We also provide preliminary theoretical analysis for these quantum phase transitions.
\end{abstract}

DOI: 10.1103/PhysRevX.9.021022

Subject Areas: Condensed Matter Physics, Particles and Fields

\section{INTRODUCTION}

The interplay between lattice gauge theories and fermionic matter has allured the imagination of physicists for

\footnotetext{
*wanderxu@gmail.com

†iyang@fudan.edu.cn

zymeng@iphy.ac.cn
}

Published by the American Physical Society under the terms of the Creative Commons Attribution 4.0 International license. Further distribution of this work must maintain attribution to the author(s) and the published article's title, journal citation, and DOI. several decades [1-15]. This is because gauge fields coupled to matter fields is a fundamental concept in many areas of physics. For example, in condensed matter, $(2+1) \mathrm{D}$ field theories with a compact $\mathrm{U}(1)$ gauge field coupled to gapless relativistic fermions often serve as the low-energy effective field theories in 2D strongly correlated electron systems including cuprate superconductors $[1,2$, 5-7] and quantum spin liquids [11-14,16]. In high-energy physics, the mechanism of quark confinement in gauge theories with dynamical fermions such as quantum chromodynamics (QCD) is among the most elusive subjects, and the absence or presence of a deconfined phase in $3 \mathrm{D}$ compact quantum electrodynamics $\left(\mathrm{cQED}_{3}\right)$ coupled to 
(not necessarily large) $N_{f}$ massless fermions has attracted a lot of attention [4,10-15] and remains unsolved to this day.

In recent years, collective efforts from both condensed matter and high-energy physics have started to generate promising outcomes $[4,12,17-26]$. There exist concrete examples, by now, of discrete $Z_{2}$ gauge field theories coupled to fermionic matter at $(2+1) \mathrm{D}$, deconfined phase with fractionalized fermionic excitations at weak gauge fluctuation, as well as symmetry-breaking phase with gapped fermionic excitations at strong gauge fluctuation have been observed [18-20]. The apparently continuous transition between the deconfined and confined phases is highly nontrivial [20], as it is driven by the condensation of emergent fractionalized excitations and is hence beyond the scope of the Landau-Ginzburg-Wilson paradigm of critical phenomena in which symmetry breaking is described by a local order parameter.

The $\mathrm{CQED}_{3}$ is the simplest theory to discuss confinement and chiral symmetry breaking [27-29]. The pure $\mathrm{CQED}_{3}$ without a matter field is known to be always confining $[10,27,30,31]$. However, when there is fermionic matter, the gapless fermionic fluctuations may drive the system towards deconfinement. The large- $N_{f}$ limit of $\mathrm{CQED}_{3}$ with fermionic matter is believed to belong to this case $[11,13,15,32]$, but the existence of the deconfined phase for small $N_{f}$ is still under debate [12,17,33-36]. Analytically, the perturbative calculation at small $N_{f}$ is uncontrolled. Numerically, recent hybrid Monte Carlo (HMC) calculations in Ref. [17] faced difficulties caused by fermion zero modes. Though these difficulties may be cured by turning on a four-fermion interaction term, the scaling dimension of the four-fermion interaction will receive corrections from gauge fluctuation at the order of $1 / N_{f}$, which may lead to a relevant runaway flow in renormalization group (RG) calculation [37]. Thus, a combined RG flow of monopole and four-fermion interactions may be complicated, and a deconfined phase could still exist in the phase diagram but evades the previous study of HMC. $\mathrm{CQED}_{3}$ with finite $N_{f}$ flavors of fermionic matter is particularly important to condensed matter physics because these cases actually correspond to the low-energy field theory description of many interesting strongly correlated electron systems, and therefore host the potential promise of establishing the new paradigms in condensed matter physics. Furthermore, perturbative renormalization-group calculations to higher orders have recently been carried out in attempt to acquire the critical properties of the deconfinement-to-confinement transition in the form of $\mathrm{QED}_{3}$-Gross-Neveu universality classes [38-41].

Based on these considerations, in this work, we succeed in performing large-scale quantum Monte Carlo (QMC) simulations on the $\mathrm{CQED}_{3}$ coupled to $N_{f}$ flavor of fermions and eventually map out the phase diagram (Fig. 1) in the fermion flavor and gauge field fluctuations strength plane. Deconfined phases- $U(1)$ deconfined phase $(\mathrm{U}(1) \mathrm{D}$ hereafter) to be more precise-are indeed found in the phase diagram for $N_{f}=6$ and 8 , and even at $N_{f}=2$ and 4 there are very positive signatures of their existence. Various confined phases in the form of different symmetry breakings, such as antiferromagnetic order (AFM) and valencebond solid (VBS), are also discovered. Interesting quantum phase transitions between deconfined and confined phases and between different confined phases [18,42-46] are revealed as well.

For the sake of a smoother narrative, the rest of the paper is organized in the following order. In Secs. II A and II B, we first start with a quantum rotor model coupled to fermions, which can be formulated as $\mathrm{CQED}_{3}$ coupled to fermionic matter. Then in Sec. II C, we discuss the sign structure of this model, where we find that a pseudounitary group can be used to avoid the phase problem at odd $N_{f}$ and the sign problem at even $N_{f}$. In Secs. II D and II E, we explain the challenges in the QMC simulation even without the sign problem and provide our solution with a fast update algorithm for simulating gauge fields with continuous symmetries. In Sec. III, we discuss the whole phase (a)

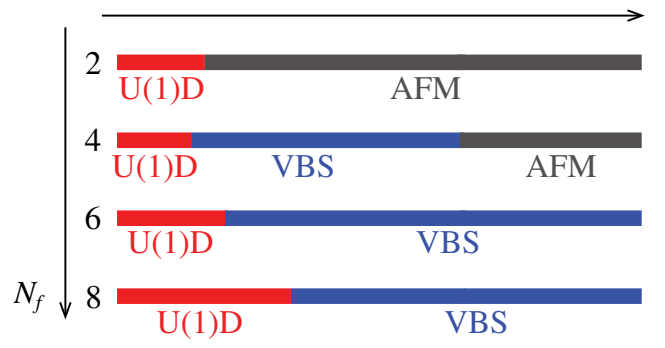

(b)

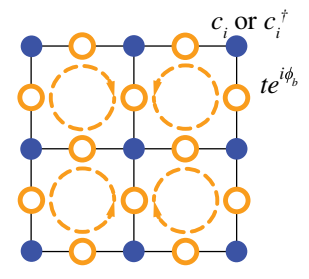

(c)

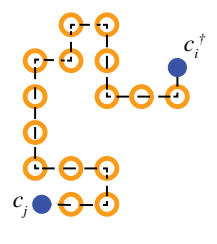

FIG. 1. (a) Phase diagram spanned by the Fermi flavors $N_{f}$ and the strength of gauge field fluctuations $J$ of the model shown in (b). $\mathrm{U}(1) \mathrm{D}$ stands for the $\mathrm{U}(1)$ deconfined phase where the fermions dynamically form a Dirac system. This phase corresponds to the algebraic spin liquid where all correlation functions show slow power-law decay. VBS stands for valence-bond-solid phase and AFM stands for the antiferromagnetic long-range ordered phase (Néel phase). (b) Sketch of the model of Eq. (1). The yellow circles represent the gauge field attached to each fermion hopping, and the yellow dashed lines stand for the flux term per plaquette. (c) The gaugeinvariant propagator for fermions with a string of gauge fields attached. 
diagram and then focus on the physical properties and understanding of the $\mathrm{U}(1) \mathrm{D}$ phase, in particular, the reason for it being the parent state of various quantum phases, the deconfinement-confinement phase transitions, and VBS-toAFM phase transition at $N_{f}=4$. Preliminary theoretical analysis of these transitions is also given in Sec. III. Finally, the discussion and conclusions are given in Sec. IV.

\section{MODEL AND METHOD}

\section{A. Rotor model with fermions}

The system we are interested in can be most conveniently formulated as a 2D quantum rotor model coupled to fermions with Hamiltonian

$$
\begin{aligned}
H= & \frac{1}{2} J N_{f} \sum_{\langle i, j\rangle} \frac{1}{4} \hat{L}_{i j}^{2}-t \sum_{\langle i, j\rangle \alpha}\left(\hat{c}_{i \alpha}^{\dagger} e^{\left.i \hat{\theta}_{i j} \hat{c}_{j \alpha}+\text { H.c. }\right)}\right. \\
& +\frac{1}{2} K N_{f} \sum_{\square} \cos (\operatorname{curl} \hat{\theta})
\end{aligned}
$$

where $\hat{L}_{i j}$ and $\hat{\theta}_{i j}$ are canonical angular momentum, $\left[\hat{L}_{i j}, e^{ \pm i \hat{\theta}_{i j}}\right]= \pm e^{ \pm i \hat{\theta}_{i j}}$, and its coordinate operator of rotors on each bond $b=\langle i j\rangle$ of a 2D square lattice, as depicted in Fig. 1(b). The fermion flavor $\alpha$ runs from 1 to $N_{f}$, and the fermions are minimally coupled to the rotor via nearestneighbor hopping on the square lattice. The flux term with $K>0$ favors $\pi$ flux in each elementary plaquette $\square$, where the magnetic flux of each plaquette $\square$ is defined as $\operatorname{curl} \hat{\theta}=\sum_{b \in \square} \hat{\theta}_{b}$, and the summation over $\hat{\theta}_{b}$ is taken in either clockwise or anticlockwise orientation around an elementary plaquette.

For the Monte Carlo simulations, it is convenient to work in a representation where $\hat{\theta}_{i j}$ is diagonal. That is, omitting the bond index $\hat{\theta}|\phi\rangle=\phi|\phi\rangle$ with $\phi \in[0,2 \pi)$. In this representation, $\hat{L}=-i[\partial /(\partial \phi)]$ with eigenvectors $\hat{L}\langle\phi \mid l\rangle \equiv \hat{L} e^{i \phi l}=l e^{i \phi l}$ and $l \in \mathbb{Z}$. With these definitions, the resolution of unity reads $\int_{0}^{2 \pi}|\phi\rangle\langle\phi|=$ $[1 /(2 \pi)] \sum_{l}|l\rangle\langle l|=\hat{1}$. To formulate the path integral, we have to estimate the matrix element: $\left\langle\phi^{\prime}\left|e^{-\Delta \tau J N_{f} \hat{L}^{2} / 8}\right| \phi\right\rangle$. To this end, we insert resolution of the unit operator and use the Poisson summation formula to obtain

$$
\left\langle\phi^{\prime}\left|e^{-\Delta \tau J N_{f} \hat{L}^{2} / 8}\right| \phi\right\rangle \sim e^{-\left[4 /\left(\Delta \tau J N_{f}\right)\right]\left[1-\cos \left(\phi-\phi^{\prime}\right)\right]},
$$

where the Villain approximation is used. With the above, the Hamiltonian in Eq. (1) can be formulated in a coherent-state path integral with action $S=S_{F}+S_{\phi}=$ $\int_{0}^{\beta} d \tau\left(L_{F}+L_{\phi}\right)$, and the Lagrangian for the fermion and gauge field parts is

$$
\begin{aligned}
L_{F}= & \sum_{\langle i j\rangle \alpha} \psi_{i \alpha}^{\dagger}\left[\left(\partial_{\tau}-\mu\right) \delta_{i j}-t e^{i \phi_{i j}}\right] \psi_{j \alpha}+\text { H.c., } \\
L_{\phi}= & \frac{4}{J N_{f} \Delta \tau^{2}} \sum_{\langle i j\rangle}\left\{1-\cos \left[\phi_{i j}(\tau+1)-\phi_{i j}(\tau)\right]\right\} \\
& +\frac{1}{2} K N_{f} \sum_{\square} \cos (\operatorname{curl} \phi),
\end{aligned}
$$

respectively, where $\mu$ is set to zero for the half-filled case. $\beta=(1 / T)$ is the inverse temperature. The model in Eq. (1) is now explicitly formulated as (unconstrained) $\mathrm{cQED}_{3}$ coupled to fermionic matter $[11,13,17]$. We now consider the symmetries of the model and show that the Gauss law is dynamically imposed in the low-temperature limit.

\section{B. Symmetries and limiting cases}

Our model [see Eq. (1)] has global and local symmetries. It enjoys a manifest global $\mathrm{SU}\left(N_{f}\right)$ spin symmetry as well as a particle-hole symmetry:

$$
\hat{P}^{-1} z \hat{c}_{i \alpha}^{\dagger} \hat{P}=\bar{z}(-1)^{i} \hat{c}_{i \alpha} .
$$

In the above, $z$ is a complex number that makes it clear that the particle-hole symmetry is antiunitary, and $(-1)^{i}$ takes the value $1(-1)$ on sublattice $A(B)$.

The local $\mathrm{U}(1)$ gauge transformation

$$
\hat{c}_{i \alpha} \rightarrow \hat{c}_{i \alpha} e^{i \varphi_{i}}, \quad \hat{\theta}_{i j} \rightarrow \hat{\theta}_{i j}+\varphi_{i}-\varphi_{j}
$$

is an invariant. The generator of this local symmetry corresponds to a local conserved charge (Gauss law)

$$
\hat{Q}_{i}=-\sum_{j} \hat{L}_{i j}+\sum_{\alpha}\left(\hat{c}_{i \alpha}^{\dagger} \hat{c}_{i \alpha}-1 / 2\right)
$$

with $\left[\hat{Q}_{i}, \hat{H}\right]=0$. In our simulations, we sample over all $\hat{Q}_{i}$ sectors, such that our Hamiltonian corresponds to an unconstrained gauge theory. As a consequence, the correlation functions of gauge-dependent quantities such as the single-particle operator are local in space but not in time: $\left\langle\hat{c}_{i \alpha}^{\dagger}(\tau) \hat{c}_{j \alpha}\right\rangle=\delta_{i, j}\left\langle\hat{c}_{i \alpha}^{\dagger}(\tau) \hat{c}_{i \alpha}\right\rangle$. Below, we argue that the Gauss law constraint is dynamically imposed in the zero-temperature limit.

At $J=\infty, \hat{L}_{i j}$ vanishes and charges are completely localized since hopping on a given bond involves excitations of the rotor mode. In this limit, charge configurations corresponding to specific values of $\hat{Q}_{i}$ are degenerate, and at any finite value of $J$ the degeneracy will be lifted. The dynamical generation of the term

$$
\hat{H}_{Q}=\sum_{i, j} K_{i, j} \hat{Q}_{i} \hat{Q}_{j}+\cdots
$$


accounts for the lifting of this degeneracy. Note that since $\left\{\hat{P}, \hat{Q}_{i}\right\}=0$, the terms containing products of odd numbers of $\hat{Q}_{i}$ 's are forbidden. The above equation defines a classical model with a finite-temperature KosterlitzThouless transition. At zero temperature, the $\hat{Q}_{i}$ are frozen in a given pattern, and the Gauss law is imposed.

For $J \rightarrow \infty$, our model maps onto an $\mathrm{SU}\left(N_{f}\right)$ quantum antiferromagnetic. We again start from the $J=\infty$ degenerate case and consider $t$ in second-order degenerate perturbation theory. As we mention above, hopping of a fermion with flavor index $\alpha$ from site $i$ to nearest-neighbor site $j$ leaves the rotor in an excited state associated with energy cost $J$. The only way to remove this excitation is for a fermion with flavor index $\alpha^{\prime}$ to hop back from site $j$ to site $i$. These processes are encoded in the $\mathrm{SU}\left(N_{f}\right)$ Heisenberg model

$$
\hat{H}_{J \rightarrow \infty} \propto-\frac{t^{2}}{J} \sum_{\langle i j\rangle}\left(\hat{D}_{i j}^{\dagger} \hat{D}_{i j}+\hat{D}_{i j} \hat{D}_{i j}^{\dagger}\right)
$$

with $\hat{D}_{i j}=\sum_{\alpha=1}^{N_{f}} \hat{c}_{i \alpha}^{\dagger} \hat{c}_{j \alpha}$. In our simulations, we have on average $N_{f} / 2$ fermions per site such that the representation of the $\mathrm{SU}\left(N_{f}\right)$ group corresponds to the antisymmetric selfadjoint representation (i.e., the Young tableau corresponding to a column of $N_{f} / 2$ boxes) [47]. On the square lattice and for even values of $N_{f}$ where the negative sign problem is absent, this model has been considered in former auxiliary field QMC simulations [12]. At $N_{f}=2$, one finds an antiferromagnetic state, and at and beyond $N_{f}=6$, a VBS state. In the large- $N_{f}$ limit, we recover the MarstonAffleck [2] saddle point accounting for dimerization. At $N_{f}=4$ and in the absence of charge fluctuations, Ref. [12] finds no compelling evidence of VBS and AFM orders when considering lattices up to $24 \times 24$. On the other hand, simulations of the corresponding SU(4) Hubbard model [48] are consistent with an AFM state in the large-U limit albeit with decreasing value of the order parameter as a function of U. In our simulations, charge fluctuations are present, and the phase diagram is consistent with AFM order in the large- $J$ limit.

At $J=0, \hat{\theta}_{i j}$ becomes a classical variable in the sense that it has no imaginary time dynamics. Even in the absence of the flux term ( $K$ term) the coupling to the fermions favors, according to Lieb's theorem [49], a $\pi$ flux per plaquette, with associated dynamically generated Dirac dispersion relation of the $\hat{c}$ fermions. The fate of this state at low values of $N_{f}$ and when gauge fluctuations are accounted for is one of the central aims of our research.

\section{Absence of the sign problem for even $N_{f}$}

To simulate the above model with the quantum Monte Carlo method, we start with the partition function
$Z=\int D(\phi, \bar{\psi}, \psi) e^{-\left(S_{\phi}+S_{F}\right)}=\int D \phi e^{-S_{\phi}} \operatorname{Tr}_{\psi}\left[e^{-S_{F}}\right]$.

As the action of the gauge field part $S_{\phi}=\int_{0}^{\beta} d \tau L_{\phi}$ with $L_{\phi}$ shown in Eq. (3) is always real (thus, its exponential is always positive), the sign structure of the Monte Carlo weight will come only from the trace over fermions. To trace out fermions, we first discretize the imaginary time $\tau=z \Delta \tau\left(z=1,2, \ldots, L_{\tau}\right)$ where $L_{\tau}$ is the total number of time slices $\left(L_{\tau} \Delta \tau=\beta\right)$. Then, performing the fermion trace, we have

$$
\operatorname{Tr}_{\psi}\left[e^{-S_{F}}\right]=\left[\operatorname{det}\left(I+\prod_{z=1}^{L_{\tau}} B_{z}\right)\right]^{N_{f}}
$$

with $S_{F}=\int_{0}^{\beta} d \tau L_{F}$ and $L_{F}$ shown in Eq. (3). After the discretization of $\beta, B_{z}=e^{-\Delta \tau V_{z}}$ with $V_{z}$ the coupling matrix for each fermion flavor [we have $N_{f}$ in total; therefore, there is power $N_{f}$ in Eq. (10)], which has only elements connecting the sites between different sublattices of the square lattice $\left(V_{z}\right)_{i j}=-t e^{i \phi_{i j}}$. We recognize that such kinds of $B_{z}$ matrices form a pseudounitary group $\mathrm{SU}(n, n)$ where $2 n$ is a dimension of $B_{z}$ (total number of sites). As proved in the Appendix $\mathrm{B}, \forall D \in \mathrm{SU}(n, n)$, $\operatorname{det}(1+D) \in \Re$ holds. Therefore, the fermion weight will always be real for all integers $N_{f}$ and, most importantly, be semi-positive-definite for all even values of $N_{f}$ such that QMC simulations can be performed. Although the current paper focuses only on the compact $\mathrm{U}(1)$ gauge fields, the $\mathrm{SU}(n, n)$ group actually allows one to add extra nonHermitian terms to the model, such as a staggered imaginary chemical potential, that are also sign-problem-free for even values of $N_{f}$. It will be very interesting to study the non-Hermitian models and their properties in the presence of gauge field fluctuations and electronic interactions in future investigations.

\section{Difficulties of the QMC simulation}

Although there is no sign problem for even $N_{f}$, the simulation of Eq. (1) is by no means simple. Earlier attempts in the high-energy community have been devoted to simulate similar models by means of the hybrid Monte Carlo [4,17,35,50] with (dynamical) mass term. Mass terms are essential to avoid divergences when calculating forces in the realm of Hamiltonian [51] and Langevin [52] dynamics. Mass terms, however, introduce biases the severity of which have to be a posteriori clarified.

On the other hand, in the condensed matter community, the determinantal QMC (DQMC) is more popular, and it usually uses local updates and the mass terms are not essential here [53-56]. However, as far as we are aware, there exists no $\mathrm{DQMC}$ simulation on $\mathrm{CQED}_{3}$ coupled to 
fermionic matter, and our work hence serves as a first attempt. As we explain below, to be able to simulate the model in Eq. (1), there are several obstacles one needs to overcome.

The most obvious obstacle is the computational complexity. For general models, the computation complexity of DQMC for one sweep is $\mathcal{O}\left(\beta N^{4}\right)$ (here, $N$ is the total number of sites) for models where a fast update is applicable, the complexity can be reduced to $\mathcal{O}\left(\beta N^{3}\right)$. The most common example is the Hubbard model with on-site interaction; when we flip a single auxiliary field at time slice $z$ and site $i$, it changes only one diagonal value in the $B_{z}$ matrix, then the new equal-time Green's function $G^{\prime}(\tau, \tau)$ can be calculated as

$$
\begin{aligned}
G^{\prime} & =\left[I+B_{1} B_{2}, \ldots, B_{z-1}(I+\Delta) B_{z}, \ldots, B_{L_{\tau}-1} B_{L_{\tau}}\right]^{-1} \\
& =G[I+\Delta(I-G)]^{-1},
\end{aligned}
$$

where $G$ is the Green's function at the previous step. As $\Delta_{i i}$ is the only nonzero element in the $N \times N$ matrix $\Delta$, $\Delta(1-G)$ can be written as the outer product of two vectors, and then the Sherman-Morrison formula can be used to reduce the complexity of calculating Eq. (11) from $\mathcal{O}\left(N^{3}\right)$ to $\mathcal{O}\left(N^{2}\right)$ [53,54]. Such an update scheme is referred as the fast update. For one sweep over all auxiliary fields [scales as $\mathcal{O}(\beta N)$ ], the scaling will be $\mathcal{O}\left(\beta N^{3}\right)$ instead of $\mathcal{O}\left(\beta N^{4}\right)$. For models with off-site interaction, e.g., in our case due to the coupling between the fermion and gauge field, usually we need to make a further Suzuki-Trotter decomposition over all bonds (assume bond $b$ connects site $i$ and $j$, and the total number of bonds is $N_{b}$ ), and the $B_{z}$ matrix is written as

$$
B_{z}=\prod_{b=1}^{N_{b}} B_{z, b}
$$

where $B_{z, b}=e^{h_{z, b}}$ and $h_{z, b}$ is an $N \times N$ matrix with only nonzero elements $B_{z, b}(i, j)=B_{z, b}(j, i)^{*}=f\left(\phi_{i j}\right)$ where $f\left(\phi_{i j}\right)=\rho\left(\phi_{i j}\right) e^{i \theta\left(\phi_{i j}\right)}$ is the complex function of auxiliary field $\phi_{i j}$ in polar form. It is obvious that only when $\theta\left(\phi_{i j}\right)=0$ or $\pi, B_{z, b}(i, j)$ will be diagonalized by an auxiliary-field-independent unitary transformation, and then the aforementioned fast update scheme applies. There are many known models that belong to this case, such as models with Heisenberg-type interaction [57-59], models with the $Z_{2}$ (bosonic) gauge field coupled to fermionic matter $[18,19,60,61]$, etc. Novel physics has been found in these models; e.g., in Ref. [58] a continuous phase transition with fermion mass generation without spontaneously breaking any symmetry was identified (similar physics was also found in the lattice QCD community [62-65]). Unfortunately, our model in Eq. (1) involves $\mathrm{U}(1)$ gauge fields, and the auxiliary field $\phi_{i j}$ is therefore a continuous variable and $\theta\left(\phi_{i j}\right)=\phi_{i j}$; thus, our model does not belong to the cases discussed above, and naively the fast update cannot be applied.

\section{E. Fast update algorithm designed for $\mathrm{U}(1)$ gauge fields}

There is indeed an alternative way to design a fast update algorithm for the model in Eq. (1). It is based on the Woodbury matrix identity that effectively generalizes the Sherman-Morrison formula to higher-rank matrices. We recognize that the new $B_{z, b}^{\prime}$ after a single update can directly be factorized into $(1+\Delta) B_{z, b}$ with

$$
\left[\begin{array}{cc}
\Delta_{i i} & \Delta_{i j} \\
\Delta_{j i} & \Delta_{j j}
\end{array}\right]=\left[\begin{array}{cc}
\left(1-e^{-i\left(\phi_{i j}-\phi_{i j}^{\prime}\right)}\right) \sinh ^{2} \Delta \tau & \left(-e^{i \phi_{i j}}+e^{i \phi_{i j}^{\prime}}\right) \sinh \Delta \tau \cosh \Delta \tau \\
\left(-e^{-i \phi_{i j}}+e^{-i \phi_{i j}^{\prime}}\right) \sinh \Delta \tau \cosh \Delta \tau & \left(1-e^{i\left(\phi_{i j}-\phi_{i j}^{\prime}\right)}\right) \sinh ^{2} \Delta \tau
\end{array}\right],
$$

and other elements of the $N \times N$ matrix $\Delta$ are zero. With such kind of structure and note that Eq. (11) still holds, $\Delta(1-G)$ can be written as product of two matrices with dimension $N \times 2$ and $2 \times N$. Therefore, we can use the generalized version of the Sherman-Morrison formula (the Woodbury matrix identity) to calculate Eq. (11), which also has complexity $\mathcal{O}\left(N^{2}\right)$.

With such a specially designed fast update, we are now ready to simulate $\mathrm{CQED}_{3}$ coupled to fermionic matter without an artificial mass term and still enjoy the $\mathcal{O}\left(\beta N^{3}\right)$ computational complexity.

\section{RESULTS}

\section{A. Physical observables}

Our model and major results are schematically summarized in Figs. 1(a) and 1(b), respectively, but before starting the discussion of QMC results, we first introduce the QMC observables that are used to characterize the symmetric and symmetry-breaking phases and their phase transitions. Since physical observables are Hermitian, we construct and measure various gauge-invariant structure factors, including spin $\chi_{S}(\mathbf{k})$ and dimer $\chi_{D}(\mathbf{k})$ structure factors. They are defined as

$$
\begin{array}{r}
\chi_{S}(\mathbf{k})=\frac{1}{L^{4}} \sum_{i j} \sum_{\alpha \beta}\left\langle S_{\beta}^{\alpha}(i) S_{\alpha}^{\beta}(j)\right\rangle e^{i \mathbf{k} \cdot\left(\mathbf{r}_{i}-\mathbf{r}_{j}\right)}, \\
\chi_{D}(\mathbf{k})=\frac{1}{L^{4}} \sum_{i j}\left(\left\langle D_{i} D_{j}\right\rangle-\left\langle D_{i}\right\rangle\left\langle D_{j}\right\rangle\right) e^{i \mathbf{k} \cdot\left(\mathbf{r}_{i}-\mathbf{r}_{j}\right)},
\end{array}
$$

where the spin operator $S_{\beta}^{\alpha}(i)=c_{i \alpha}^{\dagger} c_{i \beta}-\left[1 /\left(N_{f}\right)\right] \delta_{\alpha \beta}$ $\sum_{\gamma} c_{i \gamma}^{\dagger} c_{i \gamma}$, and the dimer operator $D_{i}=\sum_{\alpha \beta} S_{\beta}^{\alpha}(i) S_{\alpha}^{\beta}(i+\hat{x})$ 
is defined as the dimer along the nearest-neighbor bond in the $\hat{x}$ direction.

From these structure factors, one can further construct dimensionless quantities - the correlation ratio [66] - to determine the precise position of the phase transitions. If one would like to detect the transition towards antiferromagnetic long-range order, the antiferromagnetic correlation ratio is

$$
r_{\mathrm{AFM}}=1-\frac{\chi_{S}(\mathbf{X}+\delta \mathbf{q})}{\chi_{S}(\mathbf{X})},
$$

where $\mathbf{X}=(\pi, \pi)$ is the order wave vector for the AFM on the square lattice, and $\delta \mathbf{q}=\{[(2 \pi) / L], 0\}$ is the smallest momentum away from $\mathbf{X}$. In the same vein, we define the correlation ratio for the VBS order from the dimer structure factor

$$
r_{\mathrm{VBS}}=1-\frac{\chi_{D}(\mathbf{M}+\delta \mathbf{q})}{\chi_{D}(\mathbf{M})},
$$

where $\mathbf{M}=(\pi, 0)$ is the order wave vector for the VBS. Other quantities, such as the energy density and various correlation functions (spin, dimer) in real space, are also measured in the QMC simulation.

\section{B. Phase diagram}

Now we can discuss the results from the QMC simulation of Eq. (1). Starting with the final phase diagram that schematically summarizes all the data, as shown in Fig. 1(a), the phase diagram is spanned along the axes of $N_{f}$ and $J$. We set $K=t=1$ as the energy unit and choose the gauge fluctuation strength $J$ as the tuning parameter to study different $N_{f}$ cases. For each $N_{f}$, there are different phases and phase transitions, but there are similarities for all $N_{f}$ investigated; that is, at small $J, \mathrm{U}(1)$ deconfined phases $[\mathrm{U}(1) \mathrm{D}$ in Fig. 1(a)] are universally present in the phase diagram. This finding is highly nontrivial, as we explain in the Introduction (Sec. I), from both the highenergy physics and condensed matter physics communities, the existence of such a deconfined phase in $\mathrm{CQED}_{3}$ is still under debate due to the lack of controlled calculation at finite and small $N_{f}$ [4-15]. Our finding presented here provides the first set of concrete evidence to support the existence of this phase.

Moreover, as we further elucidate in later sections, such a deconfined phase is expected to be the algebraic spin liquid $[6-9,13,14,67]$, in which critical correlations of many competing order parameters, such as antiferromagnetic order, valence-bond-solid order, charge-density wave, and superconductivity, coexist and share the same powerlaw decay due to the $\mathrm{U}(1)$ gauge deconfinement and the subsequential conformally invariant, interacting fixed point $[13,14]$. Starting from the algebraic spin liquid phase, one can easily apply various perturbations and drive the system into various symmetry-breaking phases. Therefore, the algebraic spin liquid phase, i.e., U(1)D discovered in this work, actually serves as the original state of many interesting quantum phases, hence, dubbed the "parent state of quantum phases." The discovery of such a deconfined phase is the most important result of this work.

As $J$ increases, the system goes through deconfinedconfined phase transitions to various symmetry-breaking phases. In the case of $N_{f}=2$, the symmetry-breaking phase is the AFM (Néel) phase, whereas in the case of $N_{f}=4$, the symmetry-breaking phases are the VBS and AFM phases. In addition, for further increases of $N_{f}$, the symmetry-breaking phases are solely VBS. According to Ref. [20], the deconfined-confined phase transition could be a version of the deconfined quantum critical point with emergent continuous symmetry, and in addition to the QMC data, we also provide a preliminary field theoretical description of this transition. The transition from the VBS to AFM phases inside the confined regime at $N_{f}=4$, if it is indeed continuous as our data suggest, is also a deconfined quantum critical point $[18,42-46,68,69]$ whose theory is explained later.

Overall, the presence of the $\mathrm{U}(1) \mathrm{D}$ deconfined phase and the phase transitions between the deconfined to confined phases and within the confined phases, are all intriguing and show the rich physics behind the simple model of Eq. (1).

Below, we discuss some exotic aspects of the phases and phase transitions we obtain.

\section{U(1)D phase and confinement transition}

$$
\text { 1. } N_{f}=2
$$

First we focus on the case $N_{f}=2$. In the static limit $(J \rightarrow 0)$, the gauge fields are frozen into a $\pi$-flux pattern per plaquette $[49,70]$ that results in a Dirac gapless dispersion relation. At finite $J$, the gauge fields fluctuate. and proliferation of monopoles of the gauge fields may drive spinons (the fermions) to confine. On the other hand, at large values of $J$, an $\mathrm{SU}\left(N_{f}\right)$ antiferromagnetic effective low-energy model in the self-adjoint antisymmetric representation emerges (see Sec. II B). At $N_{f}=2$, the ground state of this model is known to host an AFM.

Figure 2(a) shows the AFM correlation ratio $r_{\mathrm{AFM}}$ as a function of $J$ for different system sizes. Figure 2(b) shows the crossing points of pairs of adjacent system sizes, and a power-law extrapolation in $1 / L$ gives rise to an estimate of the confinement transition in the thermodynamic limit: $J_{c}=1.6(2)$. Since the correlation ratio shows no abrupt features, the transition from the deconfined phase to the AFM is more likely to be continuous. This is consistent with the flux energy per plaquette data presented in Fig. 13 in Appendix D. For the same $J$ values as considered in Fig. 2, the flux energy per plaquette does not develop a sharp change of slope. 

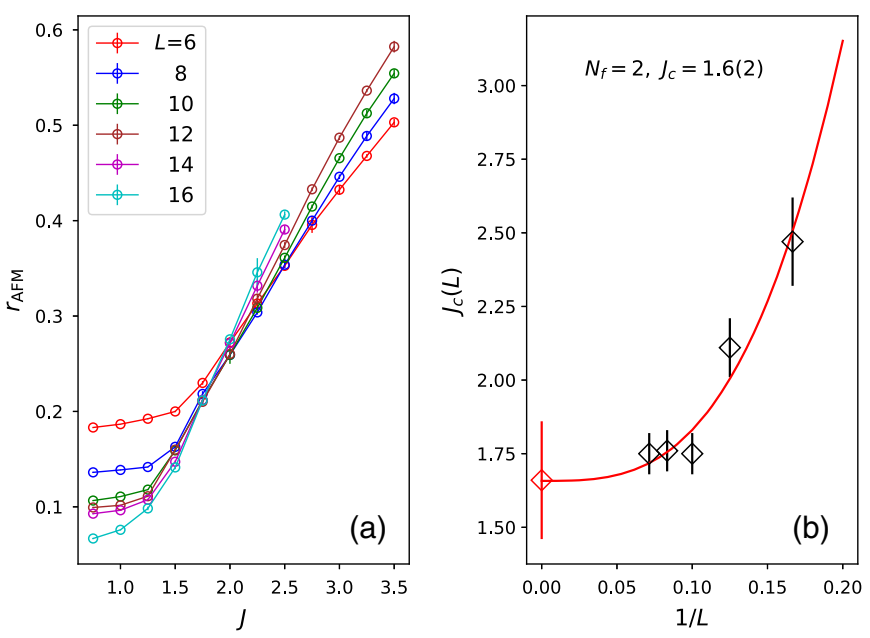

FIG. 2. (a) The antiferromagnetic correlation ratio through the $\mathrm{U}(1) \mathrm{D}$-to-AFM transition at $N_{f}=2$. Here, $\beta=4 L, \Delta \tau=0.2$. The crossing points are the transition points separating the deconfined phase and Néel phase. (b) The $1 / L$ extrapolation of the crossings estimates the U(1)D-to-AFM transition point $J_{c}=1.6(2)$ for $N_{f}=2$.

The simplest way to detect the deconfinementconfinement transition may be the Wilson loops, but it is known that in the presence of matter field, it cannot be used to detect the topological order of the deconfined phase (the U(1)D phase); such effects have been discussed in the literature [71,72]. One suitable way to demonstrate the deconfinement-confinement transition here is to show how the photon mass changes over $J$. As soon as the matter fields bind to form the particle-hole condensate, we expect monopoles to proliferate and to generate a photon mass. The photon mass can be measured by the correlation of flux quantity $\theta(\tau)$ [73], which is defined as $\theta(\tau)=\sum_{\square} \sin [\operatorname{curl} \phi(\tau)]$. The photon mass $m$ is related to the correlation of $\theta(\tau)$ by $C(\tau)=\left\langle\theta\left(\tau_{0}\right) \theta\left(\tau_{0}+\tau\right)\right\rangle \sim \exp (-m \tau)$. Figure 3 plots the estimated photon mass for different system sizes. We find a signature of an absence of photon mass in the $\mathrm{U}(1) \mathrm{D}$ phase and a growth of the photon mass in the AFM phase as $J$ increases. However, we want to point out that due to finite-size effects, i.e., uncertainties in extracting the exponential decay in $\theta(\tau)$ close to the transition, the estimation of photon mass near the phase transition is more qualitative than quantitative.

To further understand the properties of the deconfined phase, we measure the real-space correlation functions in the U(1)D phase (at $J=1.25<J_{c}$ ). As shown in Fig. 4(a), the spin-spin correlation shows a power law with the power $2 \Delta_{S}=3.1(4)\left(\Delta_{S}\right.$ is the scaling dimension of spin). Interestingly, the dimer-dimer correlation function decays with a similar power law with the power $2 \Delta_{D}=2.9(4)\left(\Delta_{D}\right.$ is the scaling dimension of the dimer) [Fig. 4(b)]. This result sheds light on the property of the deconfined phase, which is proposed in Refs. $[7,13]$ to correspond to the

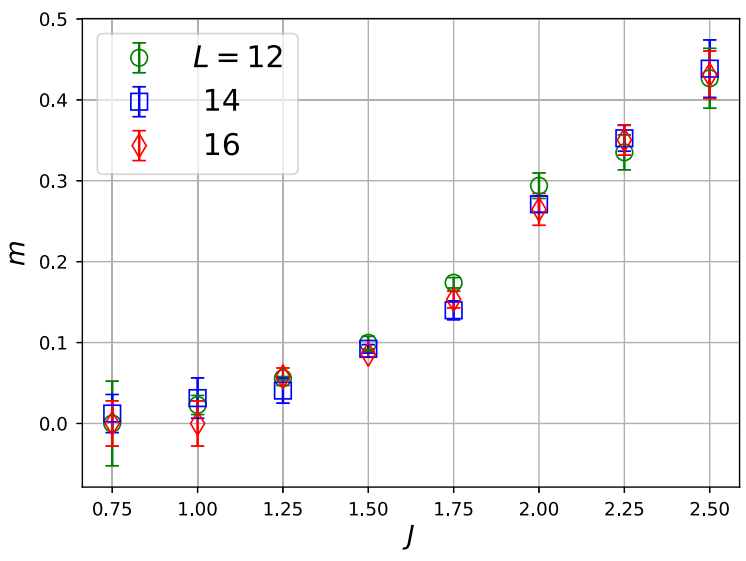

FIG. 3. Photon mass $m$ measured by the flux correlation $C(\tau)=$ $\left\langle\theta\left(\tau_{0}\right) \theta\left(\tau_{0}+\tau\right)\right\rangle \sim \exp (-m \tau) \quad$ with $\quad \theta(\tau)=\sum_{\square} \sin [\operatorname{curl} \phi(\tau)]$. Zero photon mass is observed in the $\mathrm{U}(1) \mathrm{D}$ phase; finite photon mass is observed in the confined phase. Here we plot the $N_{f}=2$ case.

algebraic spin liquid. It has the unique property that as a deconfined state emerging from competing orders, the correlation functions of these competing orders, such as spin-spin, dimer-dimer, and bond-bond, have the same power-law decay. If the data in Fig. 4 were deep inside the confined phase, the decay of spin-spin and dimer-dimer
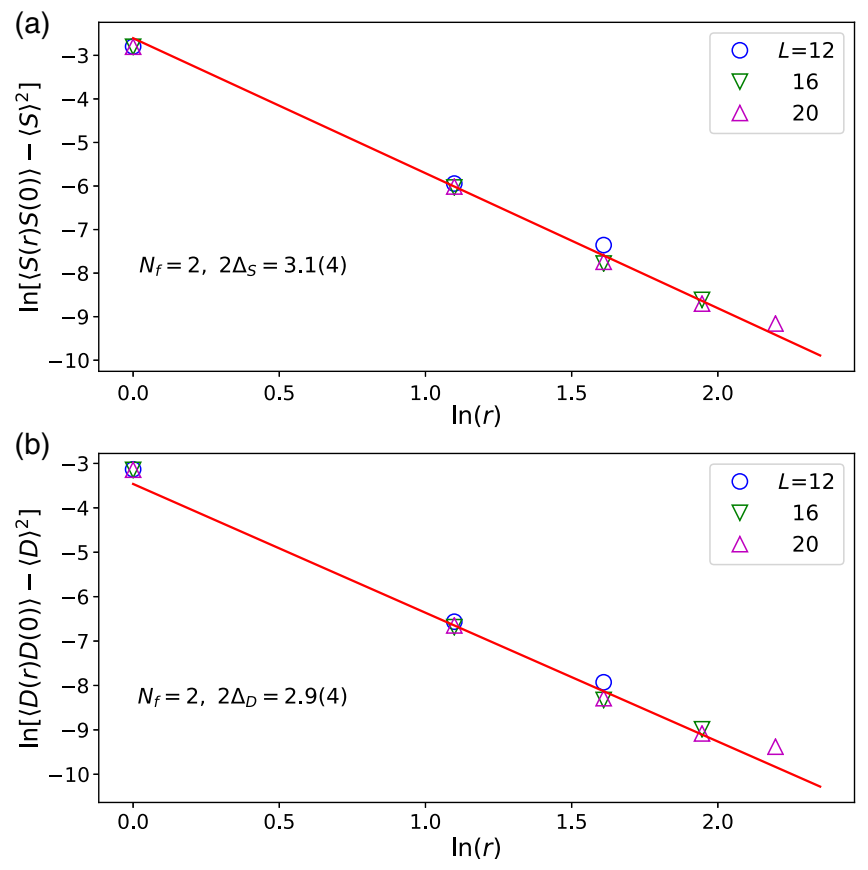

FIG. 4. The log-log plot of real-space decay of (a) spin correlation functions and (b) dimer correlation functions for $N_{f}=2$ in the $\mathrm{U}(1) \mathrm{D}$ phase (at $J=1.25<J_{c}$ ). The slope gives a good estimation of the scaling dimension of spin and dimer. Note that to avoid even-odd oscillation in the finite-size data, here only the distance $r=$ odd points are plotted in the U(1)D phase. For other $N_{f}$ cases in the following, we adopt the same strategy. 
correlations will be very different. For example, in the Néel phase, spin-spin will decay to a constant value and dimerdimer correlation will decay exponentially. Therefore, our data in Fig. 4 provide supporting evidence of the algebraic spin liquid behavior of the U(1)D in Fig. 1 at $N_{f}=2$.

\section{2. $N_{f}=4$}

Next we turn to the $N_{f}=4$ case where we also observe a $\mathrm{U}(1) \mathrm{D}$ phase at small $J$. As we show in Fig. 5(a), we can follow the crossing points of the correlation ratio of the VBS order parameter for different system sizes so as to extract [see Fig. 5(b)] $J_{c 1}=1.2(3)$. The data are consistent with a continuous phase transition from the deconfined phase to the VBS phase. Furthermore, the flux energy per plaquette also supports a continuous transition.

Figure 6 depicts the real-space decay of the spin-spin and dimer-dimer correlation functions in the $\mathrm{U}(1) \mathrm{D}$ phase for different system sizes. Again, they show similar power-law decay, and the power is estimated to be $2 \Delta_{S}=3.6(3)$ for the spin-spin correlation and $2 \Delta_{D}=3.5(3)$ for the dimerdimer correlation. This power law is faster than at $N_{f}=2$ and is hence consistent with the large- $N_{f}$ prediction [7,13,14,67].

In addition to the power-law decay of various competing correlation functions, the situation at $N_{f}=4$ is even more interesting than that at $N_{f}=2$. As we further increase $J$, we observe another phase transition from VBS to AFM. We discuss this phase transition in detail in Sec. III C 5 of the main text.

\section{Scaling dimension in the $\mathrm{U}(1) D$ phase}

The $\mathrm{U}(1) \mathrm{D}$ phase found in the small- $J$ region is expected to have the same scaling dimension for the spin $\left(\Delta_{S}\right)$ and dimer $\left(\Delta_{D}\right)$ [13]. In fact, according to the large $-N_{f}$
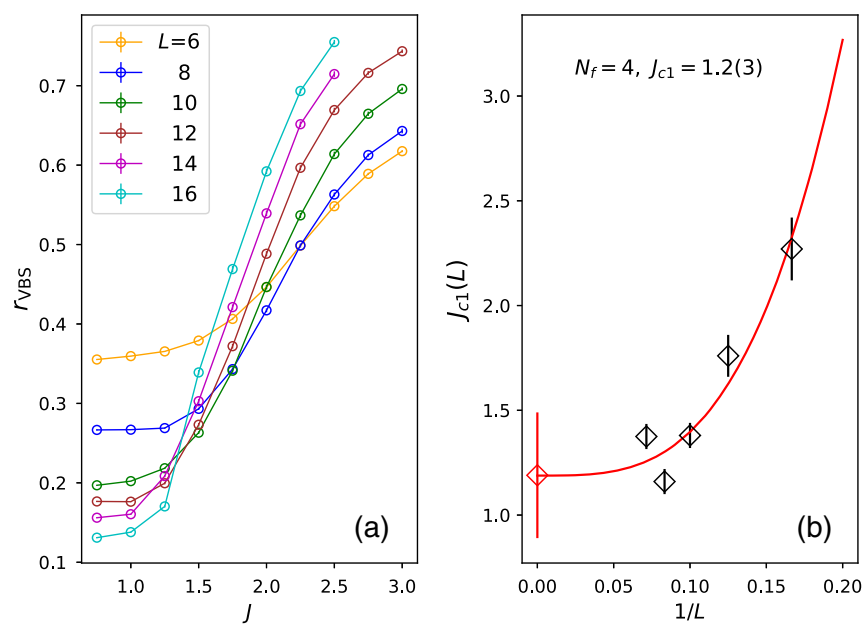

FIG. 5. The VBS correlation ratio through the U(1)D-to-VBS transition at $N_{f}=4$. Here, $\beta=3 L, \Delta \tau=0.2$. (b) The $1 / L$ extrapolation of the crossings estimates the U(1)D-to-VBS transition point $J_{c 1}=1.2(3)$ for $N_{f}=4$.
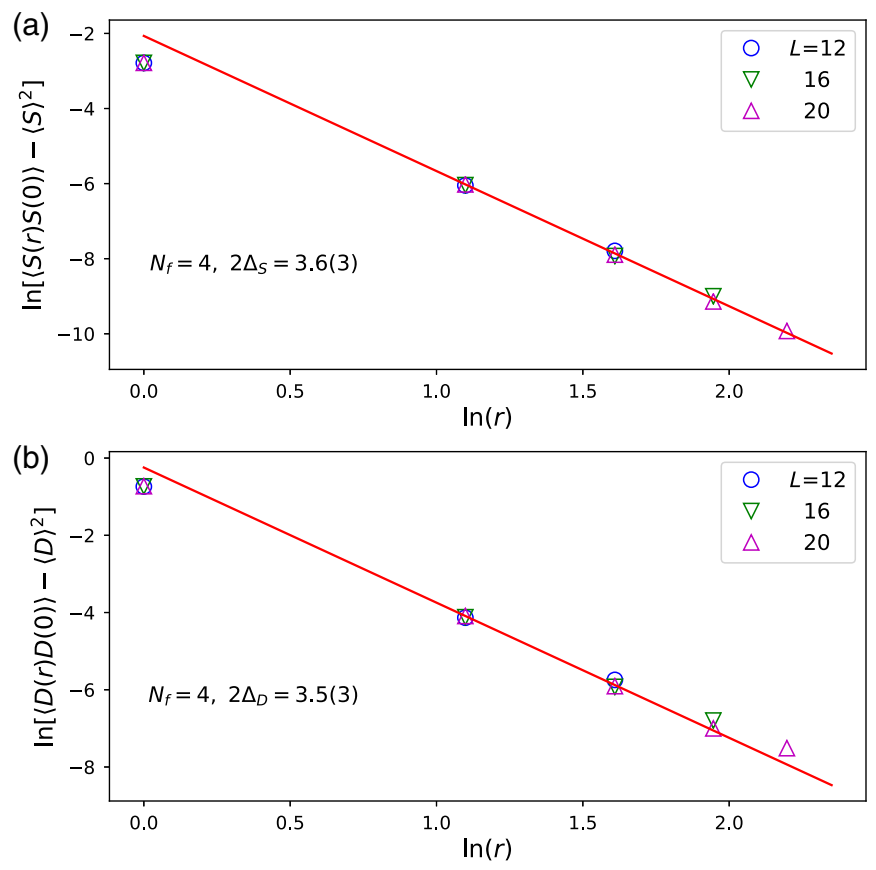

FIG. 6. The log-log plot of real-space decay of (a) spin correlation functions and (b) dimer correlation functions for $N_{f}=4$ in the $\mathrm{U}(1) \mathrm{D}$ phase (at $J=1.00<J_{c}$ ). The slope gives a good estimation of the scaling dimension of the spin and dimer.

perturbative renormalization-group calculation, these correlation functions decay as approximately

$$
r^{-\left\{4-\left[64 /\left(3 \pi^{2} N_{f}\right)\right]\right\}}
$$

with correction at $O\left(1 / N_{f}^{2}\right)[7,13,14,67]$. Notice that in our case, $N_{f}$ is the number of fermion flavors on the lattice, while in Refs. [7,13,14,67], $N_{f}$ is the number of twocomponent Dirac fermions, which is twice our $N_{f}$ due to momentum valley degeneracy.

We now compare this theoretical expectation to our numerical simulation results. Figure 7 presents a summary plot of the power law we obtain at $N_{f}=2,4,6$, and 8 . The results for $N_{f}=6$ and $N_{f}=8$ are detailed in Appendix C. It is remarkable to see that our data perfectly match the aforementioned $1 / N_{f}$ perturbative expression.

\section{Theory for confinement transition}

We find a U(1)D-to-AFM phase transition for the $N_{f}=2$ case and a U(1)D-to-VBS phase transition for the $N_{f}=4,6$, and 8 cases. These phase transitions should belong to the $\mathrm{QED}_{3}$-Gross-Neveu $\mathrm{O}(3)$ or $X Y$ transitions [38] depending on the order parameters in the confined phases. For example, at least with large enough (but still finite) $N_{f}$ when the higher-order fermion interactions are clearly irrelevant, the transition between the U(1)D to VBS phase can be described by the following action: 


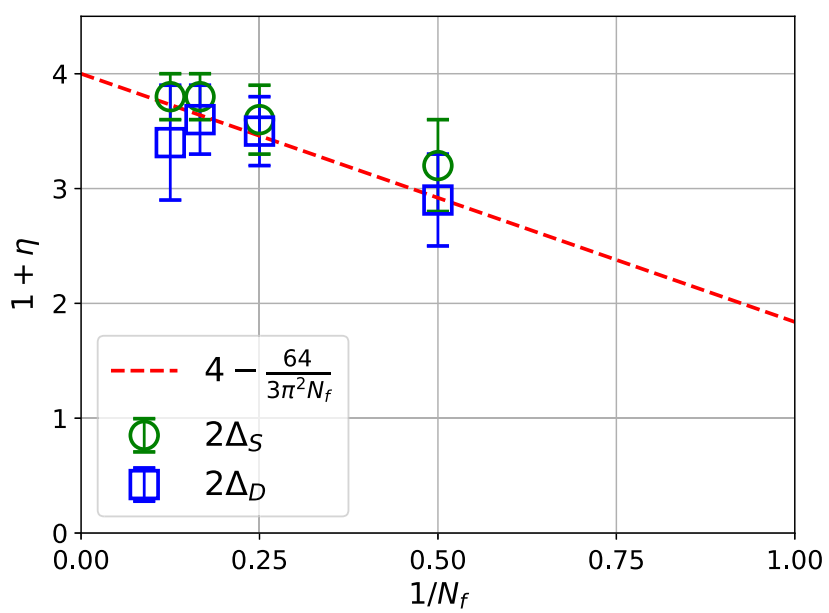

FIG. 7. Dimension of spin (gren circles) and dimer (blue squares) in the $\mathrm{U}(1) \mathrm{D}$ phase as a function of $N_{f}$. The dashed red line corresponds to the $1 / N_{f}$ perturbative calculation, $1+\eta=4-\left[64 /\left(3 \pi^{2} N_{f}\right)\right]$, taken from Ref. [7]. Note here $N_{f}$ corresponds to the number of four-component Dirac fermions.

$$
\begin{aligned}
\mathcal{S}= & \int d^{2} x d \tau \sum_{j=1}^{2 N_{f}} \bar{\psi}_{j} \gamma \cdot(\partial-i a) \psi_{j}+u \phi \cdot \bar{\psi} \mu \psi \\
& +|\partial \phi|^{2}+r|\phi|^{2}+g|\phi|^{4},
\end{aligned}
$$

where $\psi=\left(\psi_{1}, \ldots, \psi_{2 N_{f}}\right)^{T}$ has $2 N_{f}$ components, and $\phi$ is an $\mathrm{O}(2)$ vector in which the VBS order parameter is embedded. $\mu=\left(\mu^{x}, \mu^{y}\right)$ are two $2 N_{f} \times 2 N_{f}$ matrices in the fermion flavor space. $\bar{\psi} \mu^{x} \psi, \bar{\psi} \mu^{y} \psi$ are two fermion mass operators that correspond to the VBS in the $x$ and $y$ directions, respectively. When $r>0, \phi$ is gapped out, and the system is in the $\mathrm{U}(1) \mathrm{D}$ phase due to the screening of massless fermions to the gauge field. When $r<0, \phi$ condenses, the fermions are gapped out, then the compact gauge field is in the confined phase. In the U(1)D phase, the VBS and antiferromagnetic order parameters should have the same scaling dimension due to the enlarged $\mathrm{SU}\left(2 N_{f}\right)$ symmetry in the low-energy field theory, consistent with our data in Figs. 4, 6, 10, and 11, while at the critical point $r=0$, these two order parameters still have power-law correlation functions but with different scaling dimensions due to the loss of the $\mathrm{SU}\left(2 N_{f}\right)$ symmetry in the infrared.

\section{AFM-VBS transition at $N_{f}=4$}

The situation at $N_{f}=4$ is even more interesting. As we further increase $J$, we observe another quantum phase transition from the VBS to the AFM phase. This transition is consistently revealed in three steps in Fig. 8.

Figure 8(a) shows the $r_{\mathrm{AFM}}$ correlation ratio, and clearly there is a crossing point signifying the establishment of the AFM long-range order. The inset shows the $1 / L$ extrapolation of the crossing point and gives rise to $J_{c 2}=18(3)$. (a)

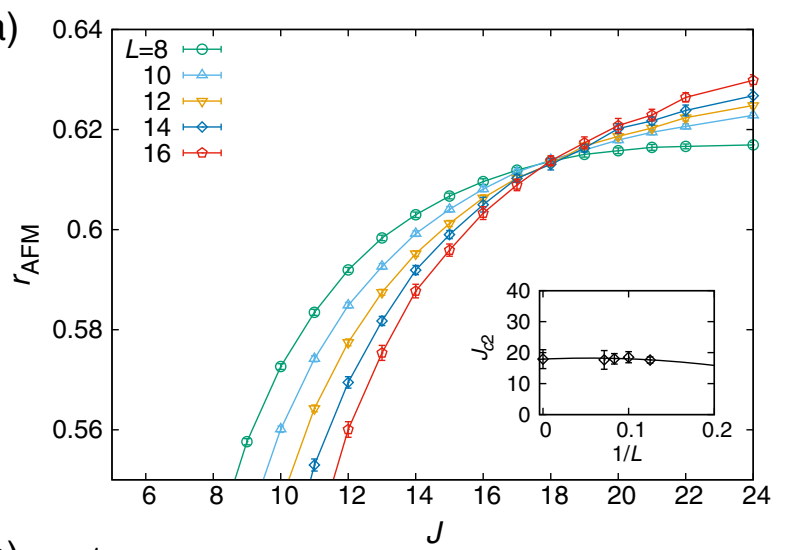

(b)

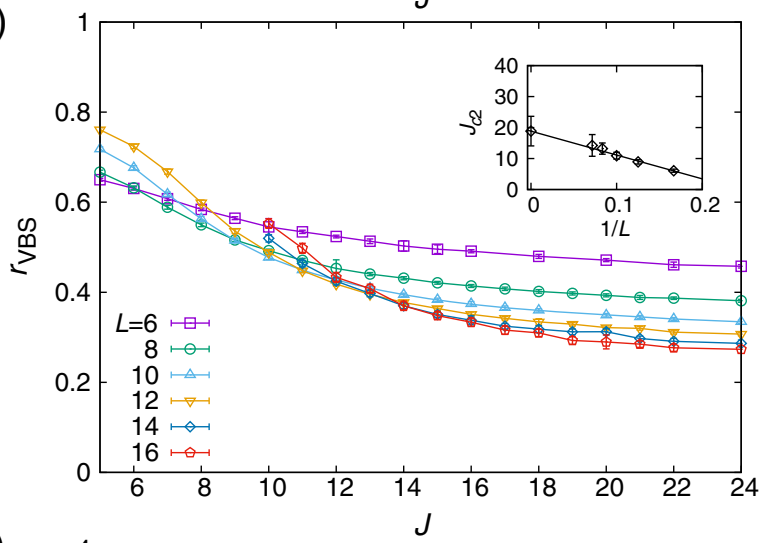

(c)

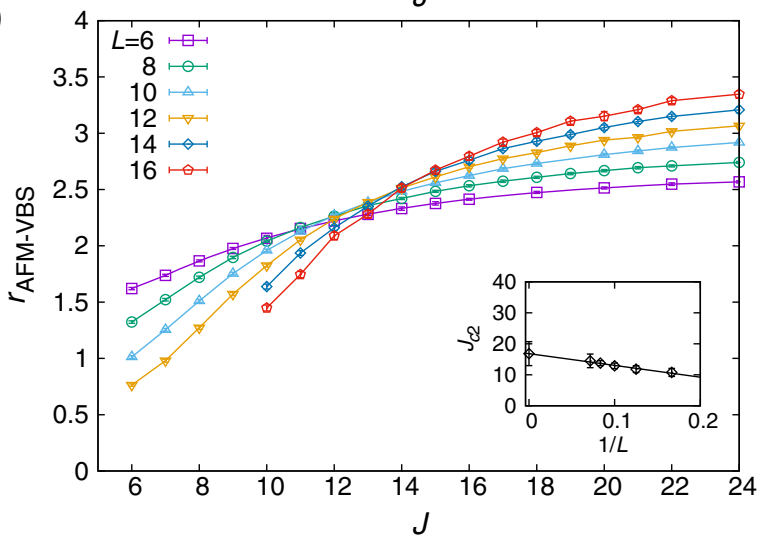

FIG. 8. (a) Antiferromagnetic correlation ratio $r_{\mathrm{AFM}}$ for $N_{f}=4$. Here, $\beta=3 L, \Delta \tau=0.2$. Insets show the $1 / L$ extrapolation of the crossing point in $r_{\mathrm{AFM}}$ and $J_{c 2}=18(3)$. (b) VBS correlation ratio $r_{\mathrm{VBS}}$ for $N_{f}=4$. The inset is the $1 / L$ extrapolation of the crossing point in $r_{\mathrm{VBS}}$ and $J_{c 2}=19(5)$. This is consistent with $J_{c 2}$ obtained from $r_{\mathrm{AFM}}$ in (a). (c) AFM-VBS correlation ratio $r_{\mathrm{AFM}-\mathrm{VBS}}$ for $N_{f}=4$. The inset is the $1 / L$ extrapolation of the crossing point in $r_{\mathrm{AFM}-\mathrm{VBS}}$ and $J_{c 2}=17(4)$.

Figure 8 (b) is the $r_{\mathrm{VBS}}$ correlation ratio, and the crossing point in it signifies the vanishing of the VBS order. The inset of Fig. 8(b) gives rise to $J_{c 2}=19(5)$, consistent with the onset of the AFM order in Fig. 8(a).

The transition from VBS to AFM deserves more attention; apparently the data in Fig. 8 suggest a continuous transition, and if it were the case, there is then the possibility that the critical point will acquire a larger 
symmetry group than that in the model in Eq. (1). In the case of the $Z_{2}$ gauge field coupled to the fermion, as shown in Refs. [18,20], two similar situations with emergent continuous symmetry are also investigated. In the first case, it is at $N_{f}=3$ that a continuous VBS-to-AFM phase transition occurs [18], and in the second case, it is the deconfinement-confinement phase transition itself at $N_{f}=2$ [20]. Our phase transition at $J_{c 2}$ is closer to the former. To further understand the nature of the transition from VBS to AFM, we plot the ratio of the AFM structure factor and VBS structure factor,

$$
r_{\mathrm{AFM}-\mathrm{VBS}}=\frac{\chi_{S}(\mathbf{X})}{\chi_{D}(\mathbf{M})}
$$

The results are shown in Fig. 8(c). Indeed, there is a crossing point in the $r_{\mathrm{AFM}} \mathrm{VBS}$, and the $1 / L$ extrapolation in the inset of Fig. 8(c) gives rise to $J_{c 2}=17(4)$, very consistent with the $J_{c 2}$ obtained from the crossings of $r_{\mathrm{AFM}}$ in Fig. 8(a) and $r_{\mathrm{VBS}}$ in Fig. 8(b). This transition is similar to the AFM-to-VBS transition in the SU(4) J-Q model [74], where a continuous transition is observed. The transition in that case can be described by a noncompact $\mathrm{CP}^{N-1}$ description with $N=4$ [75], and it is shown that the monopoles are irrelevant at this fixed point [76]. Therefore, a deconfined quantum critical point [42] is realized. However, in Ref. [75], on sublattice $A$ of the lattice, there is a fundamental representation of SU(4), while on sublattice $B$, there is an antifundamental representation, which is different from our case.

In our case, with $N_{f}=4$ there is effectively a selfconjugate representation of the $\mathrm{SU}(4)$ group on every site; thus, the field theory for the AFM-to-VBS transition is different from Ref. [75]. According to Ref. [47], the AFM Néel order in this case has the following Grassmannian ground-state manifold $\mathcal{M}$ :

$$
\mathcal{M}=\frac{\mathrm{U}(4)}{\mathrm{U}(2) \times \mathrm{U}(2)}
$$

To describe this antiferromagnetic state, one can either introduce $N_{f}=4$ flavors of fermionic spinons with halffilling, or introduce two color species of bosonic spinons $z_{\alpha, a}(\alpha=1, \ldots, 4, a=1,2)$, and couple them to a $\mathrm{U}(2)$ gauge field [to describe the simplest Néel order of SU(2) spins, we need only one two component of bosonic spinon coupled with a $\mathrm{U}(2)$ gauge field, as in Ref. [42]]. The U(2) gauge constraint will guarantee that on every site there are fixed numbers of spinons, and the color space is fully antisymmetric; thus, on every site, the SU(4) spin is automatically in an antisymmetric selfconjugate representation. Then, the field theory for the Néel-VBS transition is

$$
\mathcal{S}=\int d^{2} x d \tau\left|\left(\partial-i a-i \sum_{l=1 \cdots 3} a^{l} \tau^{l}\right) z\right|^{2}+r|z|^{2}+\cdots,
$$

where $a_{\mu}$ and $a_{\mu}^{l}$ are gauge fields corresponding to the $\mathrm{U}(1)$ and $\mathrm{SU}(2)$ subgroups of $\mathrm{U}(2)$. Note that these gauge fields are "emergent" gauge fields, which are different from the explicit gauge field in our original simulation.

When $r<0, z_{\alpha, a}$ condenses and leads to the antiferromagnetic state with ground-state manifold $\{[\mathrm{U}(4)] /[\mathrm{U}(2) \times$ $\mathrm{U}(2)]\}$. When $r>0, z_{\alpha, a}$ is gapped out, and the gauge fields will be confined. Here we assume that the U(1) compact gauge field $a_{\mu}$ still has the quadrumonopole proliferation, which leads to the VBS phase like the original deconfined QCP theory for the SU(2) spins [42].

One of the crucial properties of the deconfined QCP is the "intertwinement" between order parameters on two sides of the transition, which can be captured by a topological term which treats the Néel and VBS order parameters on equal footing [77]. In the current case with SU(4) spin symmetry, one can also introduce a topological term that captures the intertwinement between the SU(4) Néel and VBS orders with a topological term. To do this, we need to embed both the Néel and VBS order parameters into a larger manifold. One way to parametrize the Néel order manifold is $\mathcal{N}=U^{\dagger} \Omega U$, where $\Omega$ is a $4 \times 4$ diagonal matrix $\Omega=\operatorname{diag}\left(\mathbf{1}_{2 \times 2},-\mathbf{1}_{2 \times 2}\right)$, and $\mathrm{U}$ is an $\mathrm{SU}(4)$ matrix. $\mathcal{N}$ is a $4 \times 4$ Hermitian matrix with constraint $\mathcal{N}^{2}=1$.

Now we introduce a larger $8 \times 8$ matrix $\mathcal{P}$ which includes both the Néel and VBS order parameters:

$$
\mathcal{P}=\cos (\theta) \mathcal{N} \otimes \tau^{z}+\sin (\theta) \mathbf{1}_{4 \times 4} \otimes\left(V_{x} \tau^{x}+V_{y} \tau^{y}\right),
$$

where $\left(V_{x}, V_{y}\right)$ is a two-component order parameter for the VBS phase, and $V_{x}^{2}+V_{y}^{2}=1$. The order parameter $\mathcal{P}$ unifies the SU(4) Néel and VBS order parameters, just like the $\mathrm{O}(5)$ vector order parameter introduced in Ref. [77].

The topological term that captures the intertwinement between Néel and VBS order parameters is a WessZumino-Witten (WZW) term:

$$
\mathcal{S}_{\mathrm{WZW}} \sim \int d^{2} x d \tau \int_{0}^{1} d u \epsilon_{\mu \nu \rho \sigma} \operatorname{tr}\left[\mathcal{P} \partial_{\mu} \mathcal{P} \partial_{\nu} \mathcal{P} \partial_{\rho} \mathcal{P} \partial_{\sigma} \mathcal{P}\right]
$$

Using the same technique in Ref. [78], one can show that at the vortex core of the VBS order parameter, there is a spinon with self-conjugate representation, which is consistent with intuition. In fact, the $\mathrm{O}(5) \mathrm{WZW}$ term introduced in Ref. [77] can be written in the same form as Eq. (24), as long as we replace $\mathcal{P}$ in Eq. (24) by a $4 \times 4$ Hermitian matrix order parameter $\mathcal{P}=\mathbf{n} \cdot \boldsymbol{\Gamma}$, where $\boldsymbol{\Gamma}$ are five Gamma matrices, and $\mathbf{n}$ is the $\mathrm{O}(5)$ vector introduced in Ref. [77].

This topological term can be viewed as the low-energy effective field theory of the $\pi$-flux state of the SU(4) 
antiferromagnet, which again is described by a $\mathrm{QED}_{3}$ with eight flavors of Dirac fermions [13], but again the gauge field of this $\mathrm{QED}_{3}$ is an emergent gauge field which is different from the gauge field introduced in the original model that we simulate. The WZW term Eq. (24) can be derived by coupling the $8 \times 8$ matrix order parameter $\mathcal{P}$ to the eight flavors of Dirac fermions of the $\pi$-flux state and integrate out the fermions following the standard procedure of Ref. [79].

Our data, the crossing of $r_{\mathrm{AFM}-\mathrm{VBS}}$ in Fig. 8(c), suggest that the AFM and VBS order parameters have the same scaling dimension at this transition, which is consistent with the emergent $\mathrm{SU}(8)$ symmetry of the $\pi$-flux state of the SU(4) antiferromagnet. The large SU(8) symmetry, if it indeed exists at the AFM-VBS transition, will ensure that many other order parameters have the same scaling dimension as the AFM and VBS order parameters [13]. These order parameters will also have similar fractionalization dynamical signatures in their spectral functions as the AFM and VBS order parameters.

\section{CONCLUSIONS}

Using large-scale DQMC, we investigate the compact $\mathrm{U}(1)$ gauge field theory coupled to Dirac fermion matter fields in $(2+1) \mathrm{D}$ and variable flavor number $N_{f}$, i.e., $\mathrm{cQED}_{3}$. With our simulations, we map out the entire ground-state phase diagram in the flavor $N_{f}$ and gauge field fluctuation $J$ strength plane. Our results are summarized in Fig. 1(a).

Most importantly, signatures supporting stable U(1)D phases are discovered at $N_{f}=8$ and 6 , and evidence of the $\mathrm{U}(1) \mathrm{D}$ phase at $N_{f}=4$ and 2 is also found. The properties of the deconfined phase are consistent with the proposal of algebraic spin liquid, in which various competing orders (AFM order and VBS order, for example) all have algebraic correlation with identical power laws in real space. The decay power is found to quantitatively converge to the large- $N_{f}$ predictions $[7,13,14,67]$.

The transition between the deconfined and confined phases at various $N_{f}$ are determined using the RGinvariant correlation ratios. At $N_{f}=2$, the transition occurs between the U(1)D and AFM phases. Since the AFM corresponds to $\mathrm{O}(3)$ symmetry breaking, the critical theory should be described by the $\mathrm{QED}_{3}$-Gross-Neveu $\mathrm{O}(3)$ universality class. In contrast, at larger values of $N_{f}$ the ordered phases correspond to VBS. The dynamical generation of the two VBS mass terms is described by the $\mathrm{QED}_{3}$-Gross-Neveu $\mathrm{O}(2)$ universality class. As far as we know, the $\mathrm{QED}_{3}$-Gross-Neveu $\mathrm{O}(2)$ or $\mathrm{O}(3)$ transition have not been investigated numerically before in an unbiased simulation. It is certainly worthwhile to carefully study the critical properties of these transitions via QMC simulations further and compare with future analytical calculations. In particular, the $\mathrm{QED}_{3}$-Gross-Neveu Ising transition has been investigated recently with perturbative RG calculations [38,39].

Aside from the $\mathrm{QED}_{3}$-Gross-Neveu transitions, we find evidence for a direct and continuous transition between the AFM and VBS states in the confined region of the phase diagram at $N_{f}=4$. Since we have on average two fermions per site, we should consider the antisymmetric self-conjugate representation of the SU(4) group. We present various theoretical descriptions of this putative deconfined quantum phase transition in terms of multiflavored spinons coupled to emergent $\mathrm{U}(1)$ and $\mathrm{SU}(2)$ gauge fields. We also discuss the effective low-energy field theory with a topological term that captures the intertwinement between the magnetic and VBS orders and its connection to the $\pi$-flux state of the SU(4) spin system discussed in Ref. [13]. Numerical support for emergent symmetries is provided. In the future, measurements of the conserved current operators related with such emergent continuous symmetries [80] can be performed.

Finally, the confining phase transitions in our model, as well as the possible deconfined quantum critical point at $N_{f}=4$, will have distinct and very interesting dynamical properties in their spectral functions that can be further explored in QMC simulations. Such calculations provide experimentally accessible signatures of exotic states of matter where emergent gauge fields, fractionalized excitations, can be traced. Similar attempts have recently been applied to the deconfined quantum critical point in the pure spin model [69], emergent $Z_{2}$ spin liquid at $(2+1) \mathrm{D}$ [81], and $\mathrm{U}(1)$ spin liquid at $(3+1) \mathrm{D}$ [82] and the $Z_{2}$ counterpart of our model [18]. In the present $\mathrm{CQED}_{3}$ model, dynamical measurements in the QMC simulation plus state-of-the-art analytical continuation $[81,83,84]$ can help reveal more fundamental physical understanding of these exotic quantum phase transitions.

\section{ACKNOWLEDGMENTS}

The authors thank Lukas Janssen, Ribhu Kaul, Steven Kivelson, Thomas Lang, Srinivas Raghu, Subir Sachdev, Michael Scherer, Yi-Zhuang You, Ashvin Vishwanath, and Chong Wang for helpful discussions. F. F. A. would like to thank T. Grover and Zhenjiu Wang for discussions on related projects. X. Y.X., Y. Q., L.Z., and C.X. acknowledge the hospitality of the International Collaboration Center at Institute of Physics, Chinese Academy of Sciences. This work is finalized during the program "International Workshop on New Paradigms in Quantum Matter" at the center. X. Y.X. is thankful for the support of Hong Kong Research Grants Council (HKRGC) through C6026-16W, 16324216 and 16307117. Y. Q. acknowledges support from the Ministry of Science and Technology of China under Grant No. 2015CB921700 and from the National Science Foundation of China under Grant No. 11874115. Z. Y. M. 
acknowledges support from the National Key R\&D Program (Grant No. 2016YFA0300502), the Strategic Priority Research Program of CAS (Grant No. XDB28000000), and the National Science Foundation of China (Grants No. 11574359 and No. 11674370). F. F. A. thanks the DFG research unit FOR1807 for financial support. L.Z. is supported by the National Key R\&D Program of China (Grant No. 2018YFA0305802) and the Key Research Program of Chinese Academy of Sciences (Grants No. XDPB08-4 and No. XDB28040200). We thank the Center for Quantum Simulation Sciences in the Institute of Physics, Chinese Academy of Sciences, the Tianhe-1A platform at the National Supercomputer Center in Tianjin, and the Tianhe-2 platform at the National Supercomputer Center in Guangzhou for their technical support and generous allocation of CPU time.

\section{APPENDIX A: CONNECTION TO HIGH-ENERGY LATTICE CQED $_{3}$ ACTION}

As we discuss in the Sec. II A, after the path integral of the rotor degrees of freedom in a rotor model with a fermion in Eq. (1), the action of $\mathrm{cQED}_{3}$ coupled to fermionic matter is obtained explicitly. In the high-energy lattice $\mathrm{cQED}_{3}$ action, the Lagrangian for the pure gauge field part takes the form

$$
L_{\phi}=K_{\tau} \sum_{\langle i j\rangle}\left\{\cos \left[\phi_{i j}(\tau+1)-\phi_{i j}(\tau)\right]\right\}+K_{r} \sum_{\square} \cos (\operatorname{curl} \phi),
$$

where $K_{\tau}<0$ and $K_{r}<0$ with $\left|K_{\tau}\right|=\left|K_{r}\right|$. Compared with the Lagrangian defined in Eq. (3), we study the case $K_{\tau}<0$ and $K_{r}>0$ with $\left|K_{\tau}\right| \neq\left|K_{r}\right|$. As we can always rescale space and time to restore the Lorentz symmetry, the difference between $\left|K_{\tau}\right|=\left|K_{r}\right|$ and $\left|K_{\tau} \neq\right| K_{r} \mid$ is trivial. Actually, our model can be exactly mapped to the case of $K_{r}<0$ and the fermion hopping with a staggered phase factor as follows:

$$
\phi_{i, i+\hat{x}} \rightarrow \phi_{i, i+\hat{x}}+m_{y}(i) \pi
$$

where $m_{y}(i)$ is $1(0)$ if the $y$ coordinate of $i$ is odd (even), respectively. Therefore, our convention is equivalent to the high-energy lattice $\mathrm{cQED}_{3}$ action.

As we mention in the main text, the Dirac fermion in our model is realized because the $K_{r}$ term prefers $\pi$ flux through each plaquette, and the $\pi$ flux doubles the unit cell. Following the standard literature such as Ref. [13], if we start with $N_{f}$ flavors of one-component fermions on the lattice [like Eq. (1)], at low energy there will be $2 N_{f}$ flavors of two-component Dirac fermions.

\section{APPENDIX B: PSEUDOUNITARY GROUP $\mathrm{SU}(n, m)$ AND THE ABSENCE OF THE SIGN PROBLEM}

As we discuss in the main text, the fermion determinant for one flavor is $\operatorname{det}\left(I_{n+m}+\prod_{z=1}^{L_{\tau}} B_{z}\right)$, where $n$ and $m$ are the numbers of sites in the two sublattices, and $I_{n+m}$ denotes the $(n+m)$-dimensional identity matrix. $B_{z}=e^{h_{z}}$, where $h_{z}$ has the following structure,

$$
h_{z}=\left(\begin{array}{cc}
0_{n} & T_{z} \\
T_{z}^{\dagger} & 0_{m}
\end{array}\right)
$$

and $T_{z}$ is the hopping matrix between different sublattices.

$B_{z}$ matrices satisfy (1) $B_{z}^{\dagger} \eta B_{z}=\eta$, where $\eta=$ $\operatorname{diag}\left(I_{n},-I_{m}\right)$, and (2) $\operatorname{det} B_{z}=1$; thus, their products generate the pseudounitary group $\mathrm{SU}(n, m)$.

Theorem 1.-For any $D \in \mathrm{SU}(n, m)$, $\operatorname{det}\left(I_{n+m}+D\right) \in \mathbb{R}$.

Proof.-First, suppose that $\lambda$ is an eigenvalue of $D$, $D v=\lambda v$, then $D^{\dagger} \eta v=\lambda^{-1} \eta v$, and $D^{T} \eta v^{*}=\left(\lambda^{*}\right)^{-1} \eta v^{*}$; hence, $\left(\lambda^{*}\right)^{-1}$ is an eigenvalue of $D^{T}$ and is thus also an eigenvalue of $D$.

Denote the eigenvalues of $D$ by $\lambda_{i}, 1 \leq i \leq n+m$, then $\operatorname{det}\left(I_{n+m}+D\right)=\prod_{i}\left(1+\lambda_{i}\right)$. We then treat the eigenvalues on the unit circle and those not on the unit circle separately. For those not on the unit circle,

$$
\prod_{i,\left|\lambda_{i}\right| \neq 1}\left(1+\lambda_{i}\right)=\prod_{i,\left|\lambda_{i}\right|<1}\left(1+\lambda_{i}\right)\left[1+\left(\lambda_{i}^{*}\right)^{-1}\right]=\prod_{i,\left|\lambda_{i}\right|<1} \frac{\left|1+\lambda_{i}\right|^{2}}{\lambda_{i}^{*}}
$$

For those on the unit circle, denoting $\lambda_{i}=e^{i \theta_{i}}$, $-\pi<\theta_{i} \leq \pi$, we have

$$
\prod_{i,\left|\lambda_{i}\right|=1}\left(1+\lambda_{i}\right)=\prod_{i,\left|\lambda_{i}\right|=1} 2 \cos \left(\theta_{i} / 2\right) e^{i \theta_{i} / 2}
$$

Therefore, we find

$$
\begin{aligned}
{\left[\operatorname{det}\left(I_{n+m}+D\right)\right]^{2} } & =\left|\operatorname{det}\left(I_{n+m}+D\right)\right|^{2} \prod_{i} \frac{\lambda_{i}}{\left|\lambda_{i}\right|} \\
& =\left|\operatorname{det}\left(I_{n+m}+D\right)\right|^{2}>0,
\end{aligned}
$$

hence, $\operatorname{det}\left(I_{n+m}+D\right) \in \mathbb{R}$.

This theorem implies that for an even number of flavor fermions, the model is free of the sign problem for any hopping matrices $T_{z}$. For instance, this is true for both Abelian and non-Abelian gauge fields. 
It is easy to find an example

$$
D=\left(\begin{array}{cc}
-\sqrt{2} & 1 \\
1 & -\sqrt{2}
\end{array}\right) \in \mathrm{SU}(n, m)
$$

such that $\operatorname{det}(I+D)<0$. Therefore, the absence of the sign problem does not hold for an odd number of fermions in general. However, it does hold for models with fermions coupled to $\mathbb{Z}_{2}$ gauge fields [18-20].

\section{APPENDIX C: CONFINEMENT TRANSITION FOR $N_{f}=6$ AND $N_{f}=8$}

In this Appendix we discuss the results for $N_{f}=6$ and $N_{f}=8$.

As shown in Figs. 9 and 12 and the corresponding insets, we estimate $J_{c}=1.9(3)$ for $N_{f}=6$ and $J_{c}=2.5(1)$ for $N_{f}=8$. Again, the data are consistent with a continuous transition between the deconfined UID and confined VBS phases. Correspondingly, the flux energy per plaquette behaves as a smooth function across the critical point.

More interestingly, we plot the spin-spin and dimerdimer correlation functions in real space for $N_{f}=6$ at $J=1.4$ in Fig. 10 and for $N_{f}=8$ at $J=2.0$ in Fig. 11, respectively. In Fig. 10, the spin-spin and dimer-dimer correlation functions show the similar power-law decay with $2 \Delta_{S}=3.8(2)$ and $2 \Delta_{D}=3.6(3)$. In Fig. 11, both correlation functions decay with similar power, with $2 \Delta_{S}=3.8(2)$ and $2 \Delta_{D}=3.4(5)$. On the whole, our data provide concrete evidence that the deconfined phase in our model at various values of $N_{f}$ belong to the algebraic spin
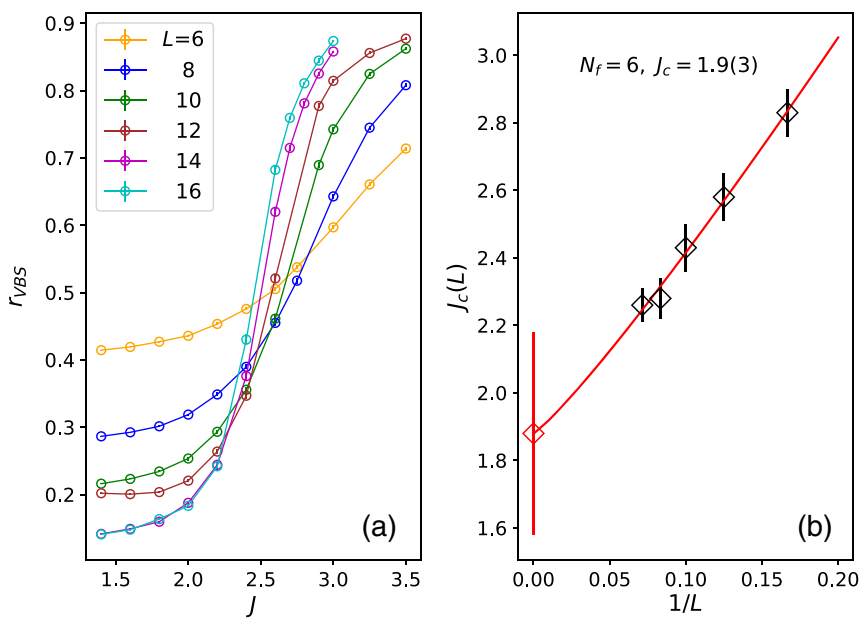

FIG. 9. The VBS correlation ratio through the U(1)D-to-VBS transition at $N_{f}=6$. Here, $\beta=2 L, \Delta \tau=0.1$. (b) The $1 / L$ extrapolation of the crossings estimates the U(1)D-to-VBS transition point at $J_{c}=1.9(3)$ for $N_{f}=6$.
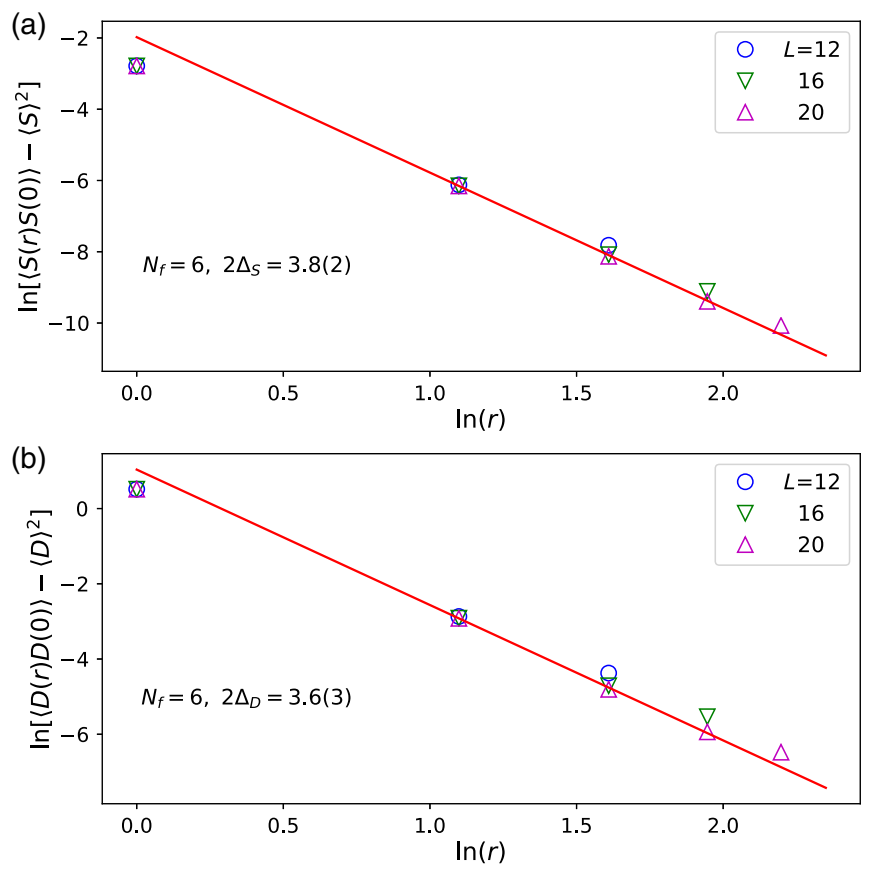

FIG. 10. The log-log plot of real-space decay of (a) spin correlation functions and (b) dimer correlation functions for $N_{f}=6$ in the $\mathrm{U}(1) \mathrm{D}$ phase (at $J=1.40<J_{c}$ ). The slope gives a good estimation of the scaling dimension of the spin and dimer.

liquid $[7,13,14,67]$. One can foresee that with a further increase of $N_{f}$, we will reach the expected power-law behavior of approximately $r^{-4}$.
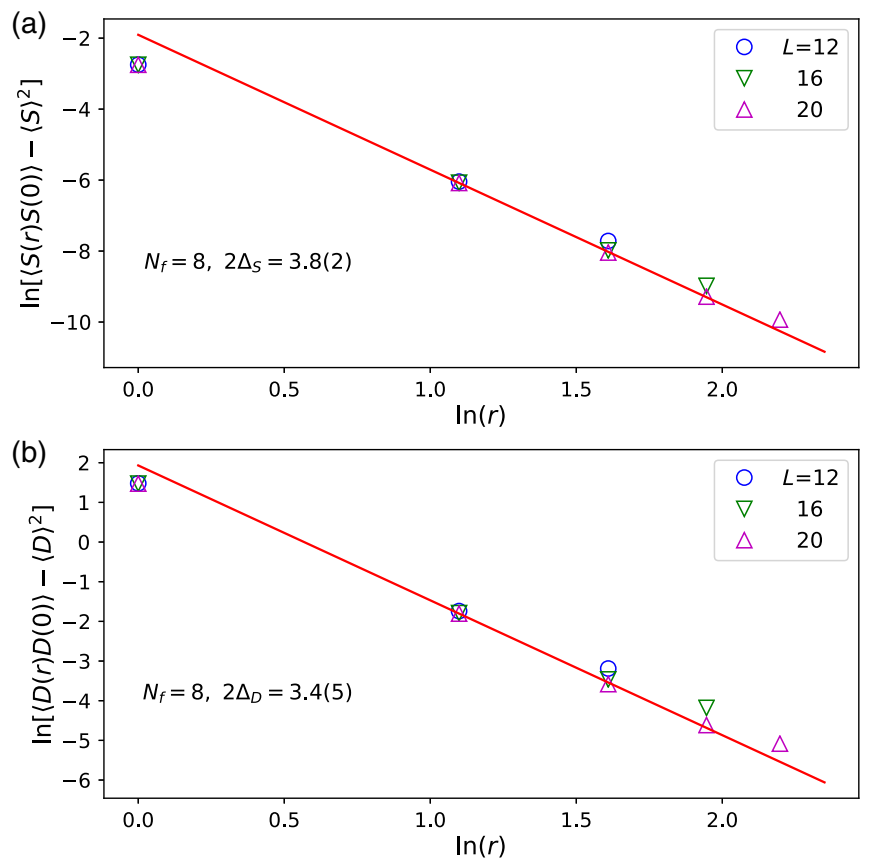

FIG. 11. The log-log plot of real-space decay of (a) spin correlation functions and (b) dimer correlation functions for $N_{f}=8$ in the $\mathrm{U}(1) \mathrm{D}$ phase (at $J=2.00<J_{c}$ ). The slope gives a good estimation of the scaling dimension of the spin and dimer. 

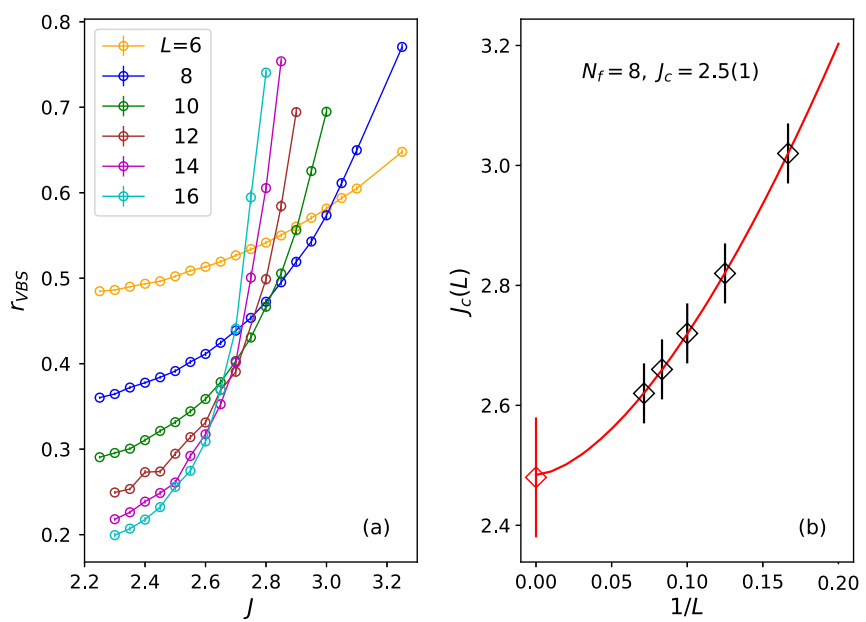

FIG. 12. The VBS correlation ratio through the U(1)D-to-VBS transition at $N_{f}=8$. Here, $\beta=2 L, \Delta \tau=0.1$. (b) The $1 / L$ extrapolation of the crossings estimates the U(1)D-to-VBS transition point at $J_{c}=2.5(1)$ for $N_{f}=8$.

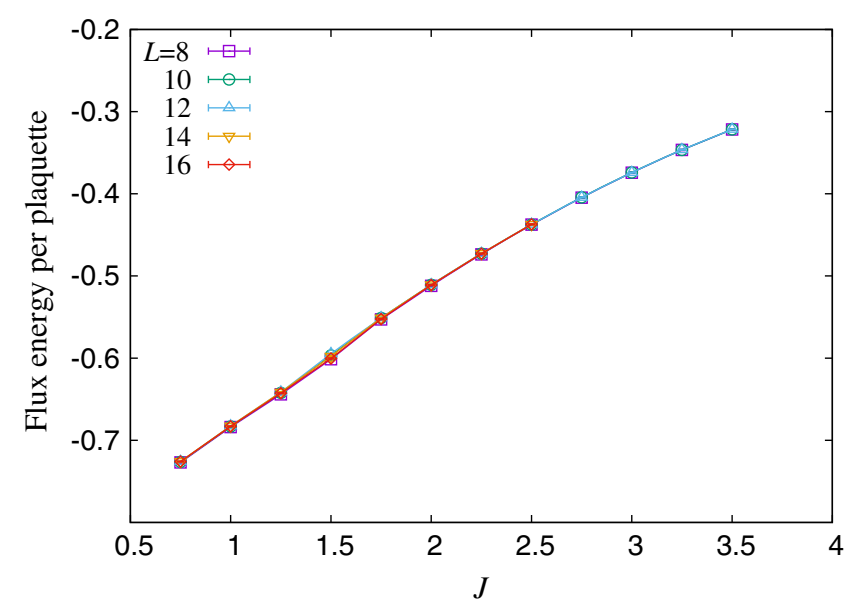

FIG. 13. Flux energy per plaquette along the same $J$ path. There is no singularity around $J_{c}$, suggesting it is a continuous phase transition.

\section{APPENDIX D: FLUX ENERGY PER PLAQUETTE}

To characterize the continuous nature of confined and deconfined phase transition, we also measure the flux energy per plaquette $\left\langle\left(1 / L^{2}\right) \sum_{\square} \cos (\operatorname{curl} \hat{\theta})\right\rangle$. Figure 13 depicts our result at $N_{f}=2$. For other $N_{f}$ 's, the flux energy per plaquette has a similar continuous behavior.

\section{APPENDIX E: DYNAMICALLY GENERATED CONSTRAINT}

As we mention in the main text, our model corresponds to an unconstrained gauge theory. As such, the Gauss law will be dynamically imposed, and $\hat{Q}_{i}$ defined in Eq. (6) should converge to constant value in the zero-temperature

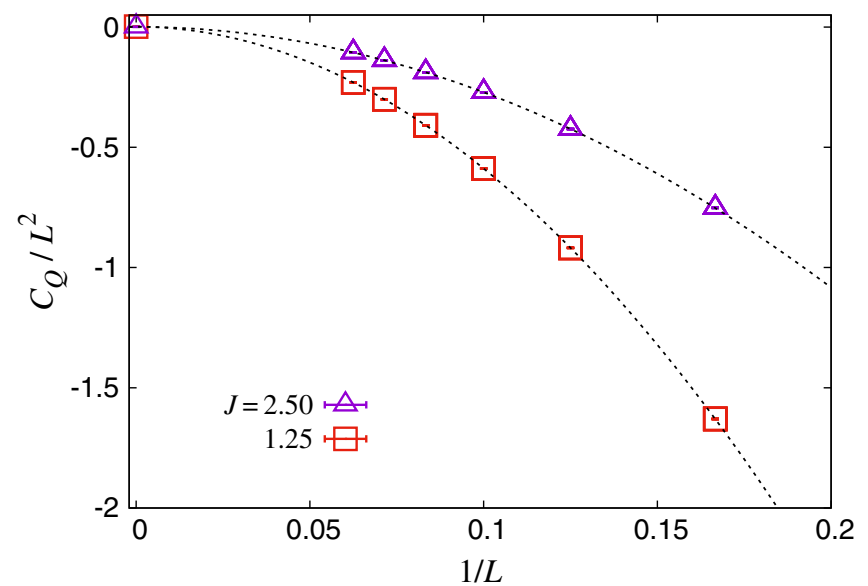

FIG. 14. Uniform structure factor of $\hat{Q}_{i}$ [defined in Eq. (E1)] for $N_{f}=2$. Note that $\beta=4 L$ here. For all $J$ 's, this uniform structure factor extrapolated to zero in thermodynamic limit. Thus, the constraint of $\hat{Q}_{i}=0$ is dynamically enforced.

limit. We study the uniform structure factor of $\hat{Q}_{i}$ by calculating

$$
C_{Q}=\frac{1}{L^{2}} \sum_{i j}\left\langle\hat{Q}_{i} \hat{Q}_{j}\right\rangle
$$

We find that the uniform structure factor of $\hat{Q}_{i}$ defined above extrapolates to zero in the thermodynamic limit as shown in Fig. 14 for $N_{f}=2$. Other $N_{f}$ cases show similar behavior.

\section{APPENDIX F: PERFORMANCE OF DQMC ON CQED $_{3}$ COUPLED TO FERMIONIC MATTER}

As we discuss in the main text, in the DQMC simulation, we use local updates which flip the gauge variables $\phi_{b}(\tau)[\in[0,2 \pi)]$ on the space-time lattice one by one, and we call one scan of the whole space-time lattice as one sweep, which is usually called one Monte Carlo step in $\mathrm{DQMC}$. For the $\mathrm{CQED}_{3}$ problem, we design a specific fast update method, which greatly improves the computation efficiency more accurately by making the fast update still work here, thus reducing the huge time cost for each sweep.

As a first attempt to study this challenging problem of $\mathrm{cQED}_{3}$ coupled to fermionic matter in the condensed matter field by the DQMC method with the local update strategy, we need to demonstrate how well it works here. The following is a detailed discussion of the performance of the method.

The first important quantity associated with the efficiency of the method is the acceptance ratio. Figure 15 illustrates the acceptance ratio for different $J$ at $N_{f}=2$. The acceptance ratio reduces as $J$ becomes smaller. Fortunately, the acceptance ratio deep in the U(1)D phase 


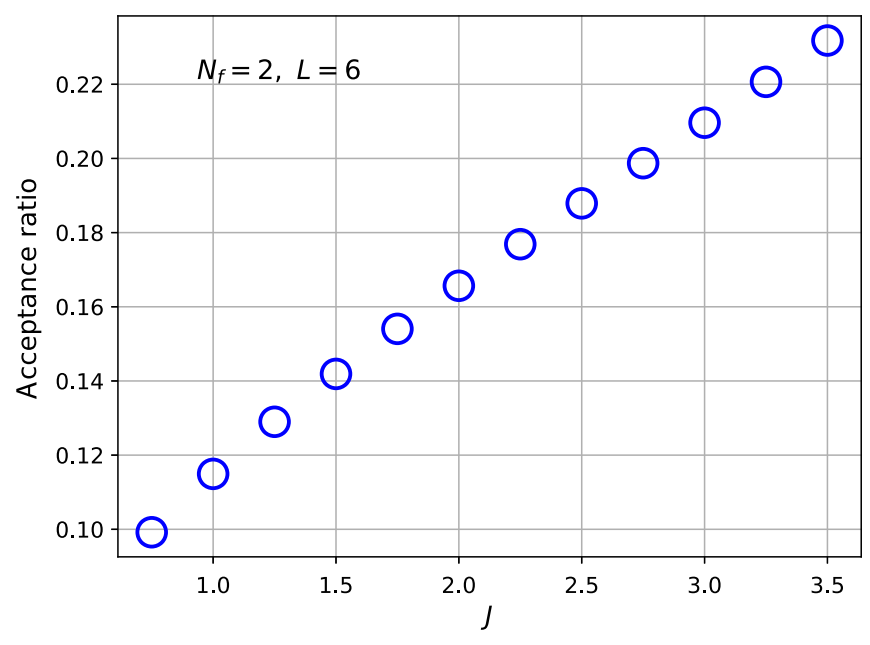

FIG. 15. Local update acceptance ratio at $N_{f}=2$ with $L=6$. Note the acceptance ratio for other sizes is almost the same, not shown here.

is still quite large; e.g., at $J=0.75<J c=1.6(2)$, the acceptance ratio is approximately $10 \%$.

The second important quantity which reflects the efficiency of our method to the specific problem we study is how quickly does the net flux change in each time plane with Monte Carlo steps? Flux in each plaquette can be written as $\sum_{b \in \square} \phi_{b}=\Phi_{\square}+2 \pi m_{\square}$ with $\Phi_{\square} \in[0,2 \pi)$ and $m_{\square}$ an integer. The net flux in one time-slice plane $M(\tau)$ is
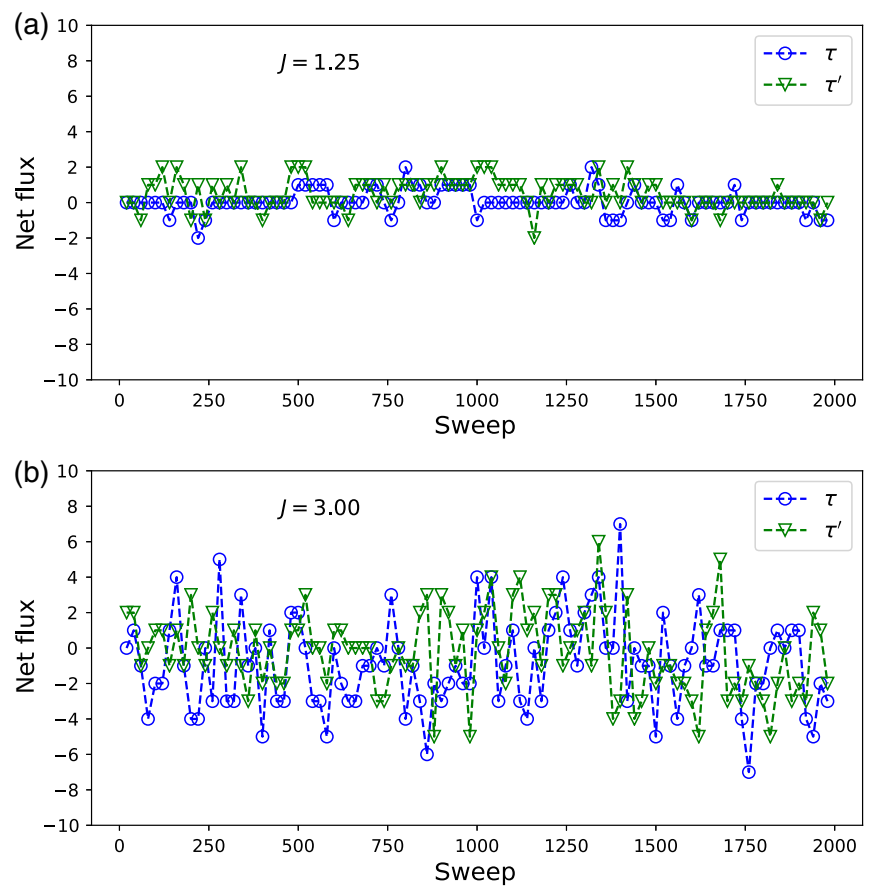

FIG. 16. Net flux sweep serials in the time-slice plane $\tau$ and $\tau^{\prime}$ at $N_{f}=2$ with $L=12$ (a) inside the U(1)D phase and (b) inside the AFM phase. Here, $\tau=\Delta \tau$ and $\tau^{\prime}=8 \Delta \tau$. The flux sweep serials are plotted in intervals of 20 sweeps, and an $L^{2} / 2$ is added to shift it to be centred around zero. defined as the sum of $m_{\square}$ of each plaquette in the time-slice plane $\tau, M(\tau)=\sum_{\square} m_{\square}(\tau)$. Figure 16 shows such net flux sweep series both inside the U(1)D phase [Fig. 16(a)] and inside the AFM phase [Fig. 16(b)] at $N_{f}=2$ with $L=12$ at different time slices $\tau$ and $\tau^{\prime}$. In the U(1)D phase, it favors the $\pi(-\pi)$ flux in each plaquette, and the net flux in each time-slice plane seldom changes and is weakly fluctuating between different time slices, while in the AFM phase, the net flux changes almost randomly with more extended values and large fluctuations between different time-slice planes, as a consequence of a proliferate of monopoles.

[1] I. Affleck and J. B. Marston, Large-n Limit of the Heisenberg-Hubbard Model: Implications for High- $T_{c}$ Superconductors, Phys. Rev. B 37, 3774 (1988).

[2] J. B. Marston and I. Affleck, Large-n Limit of the HubbardHeisenberg Model, Phys. Rev. B 39, 11538 (1989).

[3] E. Fradkin and S. Kivelson, Short Range Resonating Valence Bond Theories and Superconductivity, Mod. Phys. Lett. B 04, 225 (1990).

[4] H. R. Fiebig and R. M. Woloshyn, Monopoles and ChiralSymmetry Breaking in Three-Dimensional Lattice QED, Phys. Rev. D 42, 3520 (1990).

[5] P. A. Lee, N. Nagaosa, T.-K. Ng, and X.-G. Wen, $S U(2)$ Formulation of the $t-J$ Model: Application to Underdoped Cuprates, Phys. Rev. B 57, 6003 (1998).

[6] W. Rantner and X.-G. Wen, Electron Spectral Function and Algebraic Spin Liquid for the Normal State of Underdoped High $T_{c}$ Superconductors, Phys. Rev. Lett. 86, 3871 (2001).

[7] W. Rantner and X.-G. Wen, Spin Correlations in the Algebraic Spin Liquid: Implications for High- $T_{c}$ Superconductors, Phys. Rev. B 66, 144501 (2002).

[8] T. Senthil and M. P. A. Fisher, $Z_{2}$ Gauge Theory of Electron Fractionalization in Strongly Correlated Systems, Phys. Rev. B 62, 7850 (2000).

[9] T. Senthil and O. Motrunich, Microscopic Models for Fractionalized Phases in Strongly Correlated Systems, Phys. Rev. B 66, 205104 (2002).

[10] I. F. Herbut and B. H. Seradjeh, Permanent Confinement in the Compact $\mathrm{QED}_{3}$ with Fermionic Matter, Phys. Rev. Lett. 91, 171601 (2003).

[11] M. Hermele, T. Senthil, M. P. A. Fisher, P. A. Lee, N. Nagaosa, and X.-G. Wen, Stability of U(1) Spin Liquids in Two Dimensions, Phys. Rev. B 70, 214437 (2004).

[12] F. F. Assaad, Phase Diagram of the Half-Filled TwoDimensional SU $(N)$ Hubbard-Heisenberg Model: A Quantum Monte Carlo Study, Phys. Rev. B 71, 075103 (2005).

[13] M. Hermele, T. Senthil, and M. P. A. Fisher, Algebraic Spin Liquid as the Mother of Many Competing Orders, Phys. Rev. B 72, 104404 (2005).

[14] M. Hermele, T. Senthil, and M. P. A. Fisher, Erratum: Algebraic Spin Liquid as the Mother of Many Competing Orders [Phys. Rev. B 72, 104404 (2005)]; Erratum, Phys. Rev. B 76, 149906(E) (2007).

[15] F. S. Nogueira and H. Kleinert, Compact Quantum Electrodynamics in $2+1$ Dimensions and Spinon Deconfinement: 
A Renormalization Group Analysis, Phys. Rev. B 77, 045107 (2008).

[16] Y.-C. He, M. P. Zaletel, M. Oshikawa, and F. Pollmann, Signatures of Dirac Cones in a DMRG Study of the Kagome Heisenberg Model, Phys. Rev. X 7, 031020 (2017).

[17] W. Armour, S. Hands, J. B. Kogut, B. Lucini, C. Strouthos, and P. Vranas, Magnetic Monopole Plasma Phase in $(2+1) d$ Compact Quantum Electrodynamics with Fermionic Matter, Phys. Rev. D 84, 014502 (2011).

[18] F. F. Assaad and T. Grover, Simple Fermionic Model of Deconfined Phases and Phase Transitions, Phys. Rev. X 6 , 041049 (2016).

[19] S. Gazit, M. Randeria, and A. Vishwanath, Emergent Dirac Fermions and Broken Symmetries in Confined and Deconfined Phases of z2 Gauge Theories, Nat. Phys. 13, 484 (2017).

[20] S. Gazit, F. F. Assaad, S. Sachdev, A. Vishwanath, and C. Wang, Confinement Transition of $\mathbb{Z}_{2}$ Gauge Theories Coupled to Massless Fermions: Emergent $\mathrm{QCD}_{3}$ and $\mathrm{SO}(5)$ symmetry, Proc. Natl. Acad. Sci. U.S.A. 115, E6987 (2018).

[21] N. Karthik and R. Narayanan, Scale Invariance of ParityInvariant Three-Dimensional QED, Phys. Rev. D 94, 065026 (2016).

[22] N. Karthik and R. Narayanan, Scale-Invariance and ScaleBreaking in Parity-Invariant Three-Dimensional QCD, Phys. Rev. D 97, 054510 (2018).

[23] N. Karthik and R. Narayanan, Parity Anomaly Cancellation in Three-Dimensional QED with a Single Massless Dirac Fermion, Phys. Rev. Lett. 121, 041602 (2018).

[24] J. Braun, H. Gies, L. Janssen, and D. Roscher, Phase Structure of Many-Flavor $\mathrm{QED}_{3}$, Phys. Rev. D 90, 036002 (2014).

[25] A. V. Kotikov, V. I. Shilin, and S. Teber, Critical Behavior of $(2+1)$-Dimensional QED: $1 / N_{f}$ Corrections in the Landau Gauge, Phys. Rev. D 94, 056009 (2016).

[26] C. Prosko, S.-P. Lee, and J. Maciejko, Simple $\mathbb{Z}_{2}$ Lattice Gauge Theories at Finite Fermion Density, Phys. Rev. B 96, 205104 (2017).

[27] A. M. Polyakov, Quark Confinement and Topology of Gauge Theories, Nucl. Phys. 120B, 429 (1977).

[28] T. Banks, R. Myerson, and J. Kogut, Phase Transitions in Abelian Lattice Gauge Theories, Nucl. Phys. 129B, 493 (1977).

[29] C. D. Fosco and L. E. Oxman, Massless Fermions and the Instanton Dipole Liquid in Compact QED3, Ann. Phys. (Amsterdam) 321, 1843 (2006).

[30] S. Mandelstam, II. Vortices and Quark Confinement in NonAbelian Gauge Theories, Phys. Rep. 23, 245 (1976).

[31] M. J. Case, B. H. Seradjeh, and I. F. Herbut, Self-Consistent Theory of Compact QED3 with Relativistic Fermions, Nucl. Phys. 676B, 572 (2004).

[32] M. Unsal, Topological Symmetry, Spin Liquids and CFT Duals of Polyakov Model with Massless Fermions, arXiv:0804.4664.

[33] S. Kragset, A. Sudb $\varnothing$, and F. S. Nogueira, Metal-Insulator Transition in Two- and Three-Dimensional Logarithmic Plasmas, Phys. Rev. Lett. 92, 186403 (2004).

[34] K. Børkje, S. Kragset, and A. Sudb $\varnothing$, Instanton Correlators and Phase Transitions in Two- and Three-Dimensional Logarithmic Plasmas, Phys. Rev. B 71, 085112 (2005).
[35] R. Fiore, P. Giudice, D. Giuliano, D. Marmottini, A. Papa, and P. Sodano, $\mathrm{QED}_{3}$ on a Space-Time Lattice: Compact versus Noncompact Formulation, Phys. Rev. D 72, 094508 (2005).

[36] S. S. Pufu, Anomalous Dimensions of Monopole Operators in Three-Dimensional Quantum Electrodynamics, Phys. Rev. D 89, 065016 (2014).

[37] J. Alicea, O. I. Motrunich, M. Hermele, and M. P. A. Fisher, Criticality in Quantum Triangular Antiferromagnets via Fermionized Vortices, Phys. Rev. B 72, 064407 (2005).

[38] L. Janssen and Y.-C. He, Critical Behavior of the $\mathrm{QED}_{3}$ Gross-Neveu Model: Duality and Deconfined Criticality, Phys. Rev. B 96, 205113 (2017).

[39] B. Ihrig, L. Janssen, L. N. Mihaila, and M. M. Scherer, Deconfined Criticality from the $\mathrm{QED}_{3}$-Gross-Neveu Model at Three Loops, Phys. Rev. B 98, 115163 (2018).

[40] N. Zerf, P. Marquard, R. Boyack, and J. Maciejko, Critical Behavior of the $\mathrm{QED}_{3}$-Gross-Neveu-Yukawa Model at Four Loops, Phys. Rev. B 98, 165125 (2018).

[41] J. A. Gracey, Fermion Bilinear Operator Critical Exponents at $O\left(1 / N^{2}\right)$ in the QED-Gross-Neveu Universality Class, Phys. Rev. D 98, 085012 (2018).

[42] T. Senthil, L. Balents, S. Sachdev, A. Vishwanath, and M. P. A. Fisher, Quantum Criticality Beyond the Landau-GinzburgWilson Paradigm, Phys. Rev. B 70, 144407 (2004).

[43] A. W. Sandvik, Evidence for Deconfined Quantum Criticality in a Two-Dimensional Heisenberg Model with FourSpin Interactions, Phys. Rev. Lett. 98, 227202 (2007).

[44] Y. Q. Qin, Y.-Y. He, Y.-Z. You, Z.-Y. Lu, A. Sen, A. W. Sandvik, C. Xu, and Z. Y. Meng, Duality between the Deconfined Quantum-Critical Point and the Bosonic Topological Transition, Phys. Rev. X 7, 031052 (2017).

[45] T. Sato, M. Hohenadler, and F. F. Assaad, Dirac Fermions with Competing Orders: Non-Landau Transition with Emergent Symmetry, Phys. Rev. Lett. 119, 197203 (2017).

[46] Y.-Z. You, Y.-C. He, A. Vishwanath, and C. Xu, From Bosonic Topological Transition to Symmetric Fermion Mass Generation, Phys. Rev. B 97, 125112 (2018).

[47] N. Read and S. Sachdev, Spin-Peierls, Valence-Bond Solid, and Néel Ground States of Low-Dimensional Quantum Antiferromagnets, Phys. Rev. B 42, 4568 (1990).

[48] D. Wang, Y. Li, Z. Cai, Z. Zhou, Y. Wang, and C. Wu, Competing Orders in the $2 D$ Half-Filled $\mathrm{SU}(2 N)$ Hubbard Model through the Pinning-Field Quantum Monte Carlo Simulations, Phys. Rev. Lett. 112, 156403 (2014).

[49] E. H. Lieb, Flux Phase of the Half-Filled Band, Phys. Rev. Lett. 73, 2158 (1994).

[50] S. Duane, A. D. Kennedy, B. J. Pendleton, and D. Roweth, Hybrid Monte Carlo, Phys. Lett. B 195, 216 (1987).

[51] S. Beyl, F. Goth, and F. F. Assaad, Revisiting the Hybrid Quantum Monte Carlo Method for Hubbard and ElectronPhonon Models, Phys. Rev. B 97, 085144 (2018).

[52] G. G. Batrouni, Accelerated Langevin Simulations of Lattice QCD and Other Models, Nucl. Phys. 461A, 351 (1987).

[53] R. Blankenbecler, D. J. Scalapino, and R. L. Sugar, Monte Carlo Calculations of Coupled Boson-Fermion Systems. I, Phys. Rev. D 24, 2278 (1981).

[54] S. R. White, D. J. Scalapino, R. L. Sugar, E. Y. Loh, J. E. Gubernatis, and R. T. Scalettar, Numerical Study of the TwoDimensional Hubbard Model, Phys. Rev. B 40, 506 (1989). 
[55] F. F. Assaad and H. G. Evertz, World-line and Determinantal Quantum Monte Carlo Methods for Spins, Phonons and Electrons, in Computational Many-Particle Physics, edited by H. Fehske, R. Schneider, and A. Weiße (Springer, Berlin 2008), pp. 277-356.

[56] H. Shi and S. Zhang, Infinite Variance in Fermion Quantum Monte Carlo Calculations, Phys. Rev. E 93, 033303 (2016).

[57] Y.-Y. He, H.-Q. Wu, Y.-Z. You, C. Xu, Z. Y. Meng, and Z.-Y. Lu, Bona Fide Interaction-Driven Topological Phase Transition in Correlated Symmetry-Protected Topological States, Phys. Rev. B 93, 115150 (2016).

[58] Y.-Y. He, H.-Q. Wu, Y.-Z. You, C. Xu, Z. Y. Meng, and Z.-Y. Lu, Quantum Critical Point of Dirac Fermion Mass Generation without Spontaneous Symmetry Breaking, Phys. Rev. B 94, 241111(R) (2016).

[59] H.-Q. Wu, Y.-Y. He, Y.-Z. You, T. Yoshida, N. Kawakami, C. Xu, Z. Y. Meng, and Z.-Y. Lu, Visualizing a Bosonic Symmetry Protected Topological Phase in an Interacting Fermion Model, Phys. Rev. B 94, 165121 (2016).

[60] X. Y. Xu, K. S. D. Beach, K. Sun, F. F. Assaad, and Z. Y. Meng, Topological Phase Transitions with SO(4) Symmetry in $(2+1)$ D Interacting Dirac Fermions, Phys. Rev. B 95, 085110 (2017).

[61] Y.-Y. He, X. Y. Xu, K. Sun, F. F. Assaad, Z. Y. Meng, and Z.-Y. Lu, Dynamical Generation of Topological Masses in Dirac Fermions, Phys. Rev. B 97, 081110(R) (2018).

[62] S. Chandrasekharan, Fermion Bags and a New Origin for a Fermion Mass, arXiv:1412.3532.

[63] V. Ayyar and S. Chandrasekharan, Massive Fermions without Fermion Bilinear Condensates, Phys. Rev. D 91, 065035 (2015).

[64] V. Ayyar and S. Chandrasekharan, Fermion Masses through Four-Fermion Condensates, J. High Energy Phys. 10 (2016) 58.

[65] S. Catterall, Fermion Mass without Symmetry Breaking, J. High Energy Phys. 01 (2016) 121.

[66] S. Pujari, T. C. Lang, G. Murthy, and R. K. Kaul, Interaction-Induced Dirac Fermions from Quadratic Band Touching in Bilayer Graphene, Phys. Rev. Lett. 117, 086404 (2016).

[67] C. Xu and S. Sachdev, Square-Lattice Algebraic Spin Liquid with SO(5) Symmetry, Phys. Rev. Lett. 100, 137201 (2008).

[68] A. Nahum, P. Serna, J. T. Chalker, M. Ortuño, and A. M. Somoza, Emergent SO(5) Symmetry at the Néel to ValenceBond-Solid Transition, Phys. Rev. Lett. 115, 267203 (2015).

[69] N. Ma, G.-Y. Sun, Y.-Z. You, C. Xu, A. Vishwanath, A. W. Sandvik, and Z. Y. Meng, Dynamical Signature of
Fractionalization at a Deconfined Quantum Critical Point, Phys. Rev. B 98, 174421 (2018).

[70] A. Tanaka and X. Hu, Many-Body Spin Berry Phases Emerging from the $\pi$-Flux State: Competition between Antiferromagnetism and the Valence-Bond-Solid State, Phys. Rev. Lett. 95, 036402 (2005).

[71] M. Hermele, M. P. A. Fisher, and L. Balents, Pyrochlore Photons: The U(1) Spin Liquid in a $S=\frac{1}{2}$ Three-Dimensional Frustrated Magnet, Phys. Rev. B 69, 064404 (2004).

[72] K. Gregor, D. A. Huse, R. Moessner, and S. L. Sondhi, Diagnosing Deconfinement and Topological Order, New J. Phys. 13, 025009 (2011).

[73] A. N. Burkitt and A. C. Irving, Glueballs in 3D QED with and without Dynamical Fermions, Nucl. Phys. 295B, 525 (1988).

[74] J. Lou, A. W. Sandvik, and N. Kawashima, Antiferromagnetic to Valence-Bond-Solid Transitions in TwoDimensional $\mathrm{SU}(N)$ Heisenberg Models with Multispin Interactions, Phys. Rev. B 80, 180414(R) (2009).

[75] R. K. Kaul and A. W. Sandvik, Lattice Model for the $\mathrm{SU}(N)$ Néel to Valence-Bond Solid Quantum Phase Transition at Large N, Phys. Rev. Lett. 108, 137201 (2012).

[76] M. S. Block, R. G. Melko, and R. K. Kaul, Fate of $\mathbb{C}_{\mathbb{p}^{N-1}}$ Fixed Points with q Monopoles, Phys. Rev. Lett. 111, 137202 (2013).

[77] T. Senthil and M. P. A. Fisher, Competing Orders, Nonlinear Sigma Models, and Topological Terms in Quantum Magnets, Phys. Rev. B 74, 064405 (2006).

[78] T. Grover and T. Senthil, Topological Spin Hall States, Charged Skyrmions, and Superconductivity in Two Dimensions, Phys. Rev. Lett. 100, 156804 (2008).

[79] A. G. Abanov and P. B. Wiegmann, Theta-Terms in Nonlinear Sigma-Models, Nucl. Phys. 570B, 685 (2000).

[80] N. Ma, Y.-Z. You, and Z. Y. Meng, Emmy Noether Looks at the Deconfined Quantum Critical Point, arXiv:1811.08823 [Phys. Rev. Lett. (to be published)].

[81] G.-Y. Sun, Y.-C. Wang, C. Fang, Y. Qi, M. Cheng, and Z. Y. Meng, Dynamical Signature of Symmetry Fractionalization in Frustrated Magnets, Phys. Rev. Lett. 121, 077201 (2018).

[82] C.-J. Huang, Y. Deng, Y. Wan, and Z. Y. Meng, Dynamics of Topological Excitations in a Model Quantum Spin Ice, Phys. Rev. Lett. 120, 167202 (2018).

[83] A. W. Sandvik, Constrained Sampling Method for Analytic Continuation, Phys. Rev. E 94, 063308 (2016).

[84] H. Shao, Y. Q. Qin, S. Capponi, S. Chesi, Z. Y. Meng, and A. W. Sandvik, Nearly Deconfined Spinon Excitations in the Square-Lattice Spin-1/2 Heisenberg Antiferromagnet, Phys. Rev. X 7, 041072 (2017). 